\title{
Piercing the Milky Way: an all-sky view of the Orphan Stream
}

\author{
S. E. Koposov ${ }^{\oplus}, 1,2 \star$ V. Belokurov ${ }^{\oplus}, 2,3$ T. S. Li, ${ }^{4,5}$ C. Mateu ${ }^{\oplus},{ }^{6}$ D. Erkal ${ }^{\oplus},{ }^{7}$ \\ C. J. Grillmair, ${ }^{8}$ D. Hendel ${ }^{\odot},{ }^{9}$ A. M. Price-Whelan ${ }^{\odot},{ }^{10}$ C. F. P. Laporte, ${ }^{11} \dagger$ \\ K. Hawkins ${ }^{\circledR},{ }^{12}$ S. T. Sohn, ${ }^{13}$ A. del Pino, ${ }^{13}$ N. W. Evans, ${ }^{2}$ C. T. Slater, ${ }^{14}$ \\ N. Kallivayali1 ${ }^{15}$ and J. F. Navarro ${ }^{16} \ddagger$ \\ (The OATs: Orphan Aspen Treasury Collaboration)
}

${ }^{1}$ Carnegie Mellon University, 5000 Forbes Ave, Pittsburgh, PA 15213, USA

${ }^{2}$ Institute of Astronomy, University of Cambridge, Madingley Road, Cambridge CB3 OHA, UK

${ }^{3}$ Center for Computational Astrophysics, Flatiron Institute, 162 5th Avenue, New York, NY 10010, USA

${ }^{4}$ Fermi National Accelerator Laboratory, P.O. Box 500, Batavia, IL 60510, USA

${ }^{5}$ Kavli Institute for Cosmological Physics, University of Chicago, Chicago, IL 60637, USA

${ }^{6}$ Departamento de Astronomía, Instituto de Física, Universidad de la República, Iguá 4225, CP 11400 Montevideo, Uruguay

${ }^{7}$ Department of Physics, University of Surrey, Guildford GU2 7XH, UK

${ }^{8}$ IPAC, Mail Code 314-6, Caltech, 1200 E. California Blvd., Pasadena, CA 91125, USA

${ }^{9}$ Department of Astronomy and Astrophysics, University of Toronto, 50 St. George Street, Toronto, Ontario M5S 3H4, Canada

${ }^{10}$ Department of Astrophysical Sciences, Princeton University, 4 Ivy Lane, Princeton, NJ 08544, USA

${ }^{11}$ Department of Physics and Astornomy, University of Victoria, 3800 Finnerty Road, Victoria, BC V8P 5C2, Canada

${ }^{12}$ Department of Astronomy, The University of Texas at Austin, 2515 Speedway Boulevard, Austin, TX 78712, USA

${ }^{13}$ Space Telescope Science Institute, 3700 San Martin Drive, Baltimore, MD 21218, USA

${ }^{14}$ Department of Astronomy, University of Washington, Box 351580, Seattle, WA 98195, USA

${ }^{15}$ Department of Astronomy, University of Virginia, 530 McCormick Road, Charlottesville, VA 22904, USA

${ }^{16}$ Department of Physics and Astronomy, University of Victoria, 3800 Finnerty Road, Victoria, BC V8P 5C2, Canada

Accepted 2019 February 11. Received 2019 February 10; in original form 2018 December 20

\begin{abstract}
We use astrometry, broad-band photometry, and variability information from the Data Release 2 of ESA's Gaia mission (GDR2) to identify members of the Orphan Stream (OS) across the whole sky. The stream is traced above and below the celestial equator and in both Galactic hemispheres, thus increasing its visible length to $\sim 210^{\circ}$ equivalent to $\sim 150 \mathrm{kpc}$ in physical extent. Taking advantage of the large number of RR Lyrae stars in the OS, we extract accurate distances and proper motions across the entire stretch of the tidal debris studied. As delineated by the GDR2 RR Lyrae, the stream exhibits two prominent twists in its shape on the sky which are accompanied by changes in the tangential motion. We complement the RR Lyrae maps with those created using GDR2 Red Giants and the DECam Legacy Survey Main Sequence Turn-Off stars. The behaviour of the OS track on the sky is consistent across all three tracers employed. We detect a strong non-zero motion in the across-stream direction for a substantial portion of the stream. Such a misalignment between the debris track and the streaming velocity cannot be reproduced in a static gravitational potential and signals an interaction with a massive perturber.
\end{abstract}

Key words: Galaxy: halo-Galaxy: kinematics and dynamics-Galaxy: structure-galaxies: dwarf-galaxies: structure.
^E-mail: skoposov@cmu.edu

$\dagger$ CITA National Fellow

$\ddagger$ Senior CIfAR Fellow

\section{INTRODUCTION}

Long and narrow streams made up of stars tidally removed from low-mass satellites offer a direct, powerful and versatile means of probing the Galaxy's gravity field (see e.g. Lynden-Bell 1982; Kuhn 1993; Lynden-Bell \& Lynden-Bell 1995; Johnston, Hernquist \& Bolte 1996; Helmi \& White 1999; Johnston, Sigurdsson \& Hern- 
quist 1999; Murali \& Dubinski 1999). Discussed predominantly from a theoretical standpoint in the last decades of the past century, stellar streams started to be discovered in large numbers relatively recently (e.g. Ibata et al. 2001; Odenkirchen et al. 2001; Newberg et al. 2002; Majewski et al. 2003; Belokurov et al. 2006; Grillmair \& Dionatos 2006). Deep wide-area imaging data played a crucial role: the Sloan Digital Sky Survey (SDSS, see Gunn et al. 1998; York et al. 2000; Aihara et al. 2011; Alam et al. 2015) opened the floodgate (e.g. Grillmair 2006; Belokurov et al. 2007; Grillmair 2009; Newberg, Yanny \& Willett 2009; Koposov et al. 2012; Bonaca, Geha \& Kallivayalil 2012), and other surveys followed (e.g. Koposov et al. 2014; Bernard et al. 2016; Balbinot et al. 2016), culminating most recently with the discovery of 11 new streams in the Dark Energy Survey data (DES, see The Dark Energy Survey Collaboration 2005; Dark Energy Survey Collaboration et al. 2016) by Shipp et al. (2018).

The arrival of the Gaia data (see Gaia Collaboration et al. 2016, 2018a) has helped to push the stellar stream identification into even lower surface brightness regime through the addition of all-sky high-precision astrometry (see Myeong et al. 2018; Ibata et al. 2018; Koppelman, Helmi \& Veljanoski 2018; Malhan \& Ibata 2018; Price-Whelan \& Bonaca 2018; Belokurov \& Erkal 2019). Gaia's parallaxes and proper motions are valuable not only because they can be used to beat down the overpowering intervening Galactic foreground. The utility of a stellar tidal stream springs as soon as the kinematic information is added as demonstrated convincingly by Koposov, Rix \& Hogg (2010). For example, the streaming velocity, i.e. the component of the motion in the direction aligned with the stream, sets the normalization for the mass inside of the debris orbit, while the across-stream velocity (i.e. the velocity component tangential to the stream path) informs of possible perturbations (see e.g. Erkal et al. 2018b).

One of the first stellar streams discovered as the SDSS unleashed its data on the unsuspecting community was Orphan (see Grillmair 2006; Belokurov et al. 2007). Ten years later, the stream remains so, literally, as no plausible progenitor has been identified despite numerous attempts (e.g. Fellhauer et al. 2007; Jin \& Lynden-Bell 2007; Sales et al. 2008; Casey et al. 2013, 2014; Grillmair et al. 2015). While the stream's known length has been extended somewhat below the celestial equator, no trace of it has so far been found in the Southern Galactic hemisphere. After some initial confusion, the orbit of the stream was clarified in Newberg et al. (2010), who took advantage of the wide-area, low-resolution spectroscopy provided by the SDSS and the Sloan Extension for Galactic Understanding and Evolution (SEGUE). An equally important role was played by the blue horizontal branch (BHB) stars used by Newberg et al. (2010) to nail down the distances to the debris and thus reveal the 3-D shape of the stream. Given the distance gradient and the pattern of line-of-sight velocities, the direction of the stream motion became immediately apparent. In Galactic coordinates, the OS is moving up (as defined by the Galactic North), hence the part of the stream observed by the SDSS is leading the presumed missing section below the Milky Way's (MW) disc. By fitting orbits to the detected portions of the OS, Newberg et al. (2010) determined the stream's extrema, placing the peri-centre at $\sim 16 \mathrm{kpc}$ and the apocentre at $\sim 90 \mathrm{kpc}$.

Conveniently, not only does the OS contain plenty of BHBs but it also boasts a prominent RR Lyrae (RRL) population (see e.g. Sesar et al. 2013). In the optical, RRL are slightly better standard candles than BHB stars (see Fermani \& Schönrich 2013; Sesar et al. 2017b). Importantly, however, RRL suffer little contamination due to their unique light curves, while BHBs can be confused with Blue
Stragglers and occasionally with white dwarfs and/or QSOs (see e.g. Deason et al. 2014). An additional perk of using RRL is that a rough estimate of the star's metallicity can be obtained based on the light curve shape. Sesar et al. (2013) identified 30 RRL likely belonging to the OS using a combination of Catalina RealTime Sky Survey, Lincoln Near Earth Asteroid Research survey, and Palomar Transient Factory data. While the RRLs are scattered rather sparsely along the stream, approximately one member star per $2^{\circ}-3^{\circ}$ on the sky, they are none the less easily discernible from the bulk of the MW stellar halo. The RRL's period-luminosity relation is exceptionally tight and steep in the near-infrared. Accordingly, Hendel et al. (2018) used the Spitzer IRAC $3.6 \mu \mathrm{m}$ to follow-up the RRLs identified in Sesar et al. (2013) and measure distances to the individual OS stars with uncertainty of order $\sim 2.5$ per cent.

In this paper, we provide the first all-sky map of the Orphan Stream (OS) constructed with a range of stellar tracers. We start with the Gaia DR2 (GDR2) RRL, the largest set of RRL available to date (see Clementini et al. 2019; Holl et al. 2018). The Gaia DR2 RRL are identified and characterized in the optical where the periodluminosity relation is rather flat, thus yielding distance estimates inferior to those obtained using infrared data. None the less, they are very competitive and superior to most other tracers, with typical uncertainties $<10$ per cent. Despite the high purity (outside of the Galactic plane) and impressive completeness (for heliocentric distances below $70 \mathrm{kpc}$ ), the GDR2 RRL sample remains relatively unexplored in terms of Galactic halo studies (e.g. Iorio \& Belokurov 2019; Torrealba et al. 2018). To complement the OS RRL detections, we also study the stream with other stellar tracers, including GDR2 Red Giants (RG) and Main Sequence Turn-off (MSTO) stars in DES (Abbott et al. 2018) and DECam Legacy Survey (DECaLS, Dey et al. 2018) data.

This paper is organized as follows. Section 2 presents the details of the RRL selection from the Gaia DR2 data as well as the selection of the likely OS members. That section also gives the distributions of the OS RRL in various projections of the phase space spanned by the three spatial coordinates and two tangential velocities. Section 3 maps the stream using Gaia RG stars as well as using the matched filter technique applied to the deep DECaLS imaging. Additionally, the section shows the OS colour-magnitude diagram (CMD) and discusses the subset of the stream members with available SDSS spectroscopy. Finally. Section 4 puts the new detections of the stream into context. Concluding remarks are provided in Section 5.

\section{THE ORPHAN STREAM WITH Gaia DR2 RR LYRAE}

We select a high-purity all-sky sample of RRL candidate stars from two separate source catalogues released as part of the Gaia DR2 (Gaia Collaboration et al. 2016, 2018a). More precisely, tables vari_classifier_result and vari_rrlyrae (see Clementini et al. 2019; Holl et al. 2018) are joined, removing the duplicates, and the stellar astrometry and photometry are obtained from the main gaia_source catalogue. We provide the query for this RRL data set in the Appendix A. We have culled potential interlopers requiring phot_bp_rp_excess_factor to be less than 1.5 and have assumed $A_{\mathrm{G}} / E(B-V)=2.27$ and $M_{\mathrm{G}}=0.64$ for the extinction coefficient and the RRL absolute magnitude in the Gaia's $G$ band, respectively (see Iorio \& Belokurov 2019, for further details). We also required a negligible parallax $\varpi<\max \left(3 \sigma_{\varpi}, 0.1\right)$ and a small astrometric excess noise $(\mathrm{AEN}<1)$. Additionally, we have assumed the Sun's distance from the Galactic centre of $8.3 \mathrm{kpc}$ (Gillessen et al. 2009), the Sun's height above the Galactic mid- 
plane of $27 \mathrm{pc}$ (Chen et al. 2001), the local standard of rest of $220 \mathrm{~km} \mathrm{~s}^{-1}$ (Bovy 2015), and the Sun's peculiar motion as given in Schönrich, Binney \& Dehnen (2010).

To select the likely OS members, we translate the stellar celestial coordinates in equatorial system $(\alpha, \delta)$ into a new coordinate system $\left(\phi_{1}, \phi_{2}\right)$ aligned with the stream (see e.g. Koposov et al. 2010). More specifically, based on the OS detections reported in Belokurov et al. (2007), Grillmair (2006), and Newberg et al. (2010), we use a great circle with a pole at $\left(\alpha_{\mathrm{OS}}, \delta_{\mathrm{OS}}\right)=\left(72^{\circ},-14^{\circ}\right)$. The origin of this stream's coordinate system is chosen to be at $\left(\alpha_{0}, \delta_{0}\right)=$ (191.10487, -62.86084), near the position where the equator of this coordinate system (and the OS) crosses the Galactic plane. We provide the rotation matrix to this coordinate system in Appendix B. Finally, $\phi_{1}$ increases in the direction of the stream's motion, i.e. from the Galactic South to the Galactic North. Several attempts at the OS kinematic characterization can be found in Newberg et al. (2010) and Sohn et al. (2016). Given the OS's line-of-sight velocity and the Hubble Space Telescope-based proper motions, we notice that the stream's velocity component along Galactic longitude $v_{1}$ remains negative irrespective of the debris position on the sky, i.e. the stream is in prograde motion with respect to the Galactic rotation. Accordingly, we explore the distribution of the possible OS members selected with the following simple cuts.

$$
\begin{aligned}
\left|\phi_{2}\right| & <4^{\circ} \\
-300<\frac{v_{1}}{1 \mathrm{~km} \mathrm{~s}^{-1}} & <-100 \\
-300<\frac{v_{\mathrm{b}}}{1 \mathrm{~km} \mathrm{~s}^{-1}} & <300
\end{aligned}
$$

Fig. 1 shows the distributions of $\sim 1000$ RRL stars selected with the above cuts in Galactocentric coordinates $X, Y$, and $Z$; here $X$ points to the Galactic anticentre and the Sun is at $(X, Y, Z)=(8.3$, $0,0)$. A long and narrow arc of RRL stars is visible in all three projections, crossing the Galaxy from the North (where it is seen at $Z>0$ and $X>0$ ) to the South (where the signal is at $Z<$ 0 and $X<0$ ). The stream spans a gigantic $\sim 150 \mathrm{kpc}$, travelling in almost uninterrupted fashion through the MW, coming as close as $\sim 15 \mathrm{kpc}$ to its centre and reaching as far as $\sim 50 \mathrm{kpc}$ into the halo. Yet neither an obvious progenitor nor any sign of the stream's apocentre is apparent. While the Northern portion of the stream had been seen before with SDSS and Pan-STARRS1 (PS1; Chambers et al. 2016), the view of its Southern Galactic section uncovered here with Gaia DR2 is entirely new.

We can further clean the OS membership by looking closely at the behaviour of the RRL stars close to the equator of the stream's coordinate system. To this end, Fig. 2 shows the distribution of RRL as a function of the along-stream coordinate $\phi_{1}$. The top panel shows the distribution of RRL on the sky, the middle one shows the distance as a function of the angle along the stream, and the bottom one shows the proper motion of the stream stars along the $\phi_{1}$ direction (after correcting for the solar reflex motion). Each panel of Fig. 2 shows a sample of stars selected after applying the selection masks from the other two panels (the masked areas are highlighted in pale blue in each panel), i.e. the stars for the top panel were selected based on their proper motion and distance. An additional proper motion selection was applied to all stars in this figure to limit $-0.5<\frac{\mu_{\phi_{2}}}{1 \text { mas }^{-1}}<0.9$. The masks are specified by first adopting reference stream tracks in proper motion, distance, and on the sky. The reference stream tracks are defined as natural cubic splines. For the rest of the paper, we will refer to these spline tracks in distance, proper motion and on the sky as $\hat{D}\left(\phi_{1}\right), \hat{\mu}_{\phi, 1}\left(\phi_{1}\right)$, and $\hat{\phi}_{2}\left(\phi_{1}\right)$ The positions of the spline knots and the corresponding values were mostly chosen manually based on the RRL distribution seen on Fig. 2 and are provided in Tables C1-C3 in Appendix C. The boundaries of the masks are chosen to wrap around the reference tracks of the stream and are defined as $0.75 \hat{D}\left(\phi_{1}\right)<D<$ $1.25 \hat{D}\left(\phi_{1}\right),\left|\mu_{\phi_{1}}-\hat{\mu}_{1}\left(\phi_{1}\right)\right|<1 \mathrm{mas} \mathrm{yr}^{-1},\left|\phi_{2}-\hat{\phi}_{2}\left(\phi_{1}\right)\right|<1^{\circ}$ for distance, proper motion, and on-sky track, respectively. Applying all three masks shown in the figure yields the total of 109 OS candidate members. Table 1 lists all RRL stars identified using the procedure described above together with their positions and heliocentric distances.

As the top panel of the figure illustrates, the stream spans some $\sim 210^{\circ}$ in $\phi_{1}$, while exhibiting a slight bend of several degrees in $\phi_{2}$. The heliocentric distance of the Orphan debris changes slowly within $-50^{\circ}<\phi_{1}<50^{\circ}$, but beyond that, the stream appears to shoot rapidly to large distances, reaching $\sim 50 \mathrm{kpc}$ on either end. Finally, a clear kinematic pattern is visible in the bottom panel of Fig. 2, where the stream $\mu_{\phi, 1}$ proper motion arches up to $\sim 4 \mathrm{mas} \mathrm{yr}^{-1}$ around $\phi_{1} \sim 40^{\circ}$ from values close to $\sim 0 \mathrm{mas} \mathrm{yr}^{-1}$ near the tips of the stream. Note that in all three projections, the width of the stream also changes as a function of $\phi_{1}$. Some of this variation may be due to the intrinsic evolution of the debris density along the stream (see e.g. Erkal, Sanders \& Belokurov 2016a), but much of it may plausibly be associated with the variations in the uncertainties, especially for the proper motions.

Fig. 3 is a companion to Fig. 1 as it also shows the Galactocentric positions of the Gaia RRL stars, but now only those selected using the masks described above. The leftmost panel of Fig. 3 emphasizes the high eccentricity of the OS as the stream appears highly stretched in this projection. Noticeable in the middle and righthand panels of this figure is the change in the stream's curvature between the Southern and the North Galactic hemispheres. The view of the OS in other phase-space projections can be found in Fig. 4.

The top left panel of Fig. 4 presents the on-sky positions of the likely OS members in the stream-aligned coordinate system (note the large aspect ratio). We remark on the striking change of the stream curvature around $20^{\circ}<\phi_{1}<50^{\circ}-$ immediately after the stream re-emerges after passing through the Galactic disc - where the debris behaviour changes from gently sloping down to sharply rising up in $\phi_{2}$. Additionally, in the Northern end of the stream, at $\phi_{1}$ $\sim 100^{\circ}$ there appears to be a curious hook downwards. There exists a corresponding change in the debris kinematics. For example, proper motion components $\mu_{\alpha}$ and $\mu_{\delta}$ also show a switch in the gradient as a function of $\phi_{1}$ around $\phi_{1} \sim 20^{\circ}$. Note, however, that the evolution of the physical velocity components $v_{1}$ and $v_{\mathrm{b}}$ (and also $v_{\phi, 1}, v_{\phi, 2}, \mu_{\phi_{1}}$, and $\mu_{\phi_{2}}$ ) is considerably smoother. Therefore, most of the change in the proper motions (shown in the second row from the top) is due to the change in the line-of-sight distance and the contribution of the solar reflex motion.

One extraordinary feature observed in Fig. 4 is the strong deviation of the across-stream velocity component $v_{\phi, 2}$ when comparing the Northern part of the stream $50^{\circ}<\phi_{1}<100^{\circ}$ to the Southern extension $-50^{\circ}<\phi_{1}<0^{\circ}$. In the Southern extension - corresponding to the locations under the Galactic plane $-v_{\phi, 2} \sim 50 \mathrm{~km} \mathrm{~s}^{-1}$, while in the North $v_{\phi, 2}$ is mostly consistent with $0 \mathrm{~km} \mathrm{~s}^{-1}$. Note that in a static gravitational potential, the stars in a cold stellar stream are expected to move along the direction delineated by the stream track, i.e. with $v_{\phi, 2} \sim 0 \mathrm{~km} \mathrm{~s}^{-1}$ (see e.g. Koposov et al. 2010; Erkal et al. 2018 b). Exceptions to this rule are small regions around the location of the progenitor as well as near the apocentre. We conjecture that 

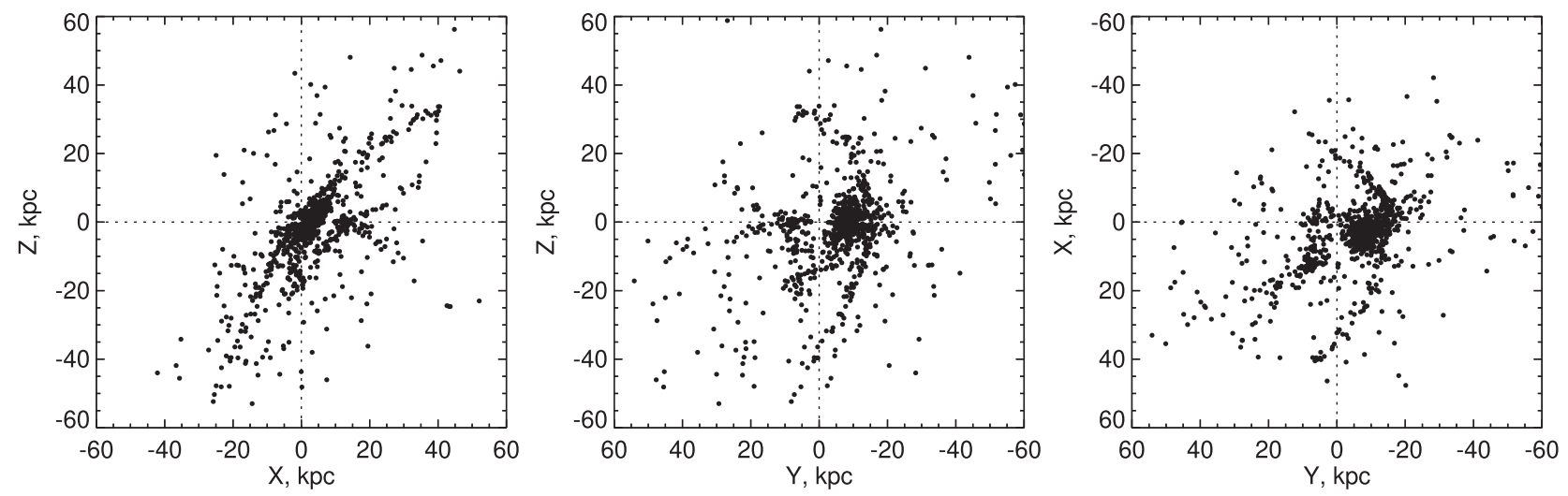

Figure 1. Galactocentric distributions of the $\sim 1000$ Gaia DR2 RRL stars projected close to the OS on the sky. Only stars with $\left|\phi_{2}\right|<4^{\circ}$ and $-300<$ $\frac{v_{1}}{1 \mathrm{~km} \mathrm{~s}^{-1}}<-100,-300<\frac{v_{\mathrm{b}}}{1 \mathrm{~km} \mathrm{~s}^{-1}}<300$ are shown. Left: $X, Z$ plane. Middle: $Y, Z$ plane. Right: $X, Y$ plane. Note a prominent, narrow, and long stream-like overdensity visible in all three panels.

the observed misalignment between the motion of the RRL and the stream path is indicative of a strong perturbation experienced by the stream in the not-so-distant past. A large and massive deflector is required to divert orbits of stars in such an extended portion of the stream.

\section{OS STARS IN GAIA, DECALS, AND SDSS}

\subsection{Track on the sky}

Guided by the RRL detections, we now chart the OS debris using other stellar tracers, while taking advantage of the stream track information in proper motion, distance, and position on the sky. As a first step we try to map the stream using the red giant branch (RGB) stars in the Gaia data. We start by identifying the OS signal in the Gaia colour-magnitude space and obtain the background subtracted Hess diagram of Orphan members. We use Gaia's $G_{\mathrm{BP}}, G_{\mathrm{RP}}$, and $G$ photometry (see Evans et al. 2018, for additional details) corrected for extinction following the prescription of Gaia Collaboration et al. (2018b). As we have measured the distance evolution along the stream from the RRL (see previous section and Table C2 for the definition of the distance spline as a function of the angle along the stream), we correct all the magnitudes of the stars by the distance modulus expected at a given $\phi_{1}$. The background subtracted Hess diagrams for the stream are constructed by selecting stars within $0.7^{\circ}$ of the stream track (as defined in Table C1) for the Northern stream and within $1^{\circ}$ for the Southern stream. The areas located between $2^{\circ}$ and $4^{\circ}$ away from the track were used as a background for the Northern stream, and $2^{\circ}$ and $6^{\circ}$ for the Southern. Additionally, we apply the proper motion selection based on splines presented in Fig. 2 and Table C3: $\left|\mu_{\phi_{1}}-\hat{\mu}_{\phi, 1}\left(\phi_{1}\right)\right|<0.5 \mathrm{mas} \mathrm{yr}^{-1}$ for both streams and $-0.5<\frac{\mu_{\phi_{2}}}{1 \text { mas yr }^{-1}}<1$ and $\left|\mu_{\phi_{2}}\right|<0.5$ mas yr $^{-1}$ for the Southern and Northern streams, respectively.

The resulting CMD for both parts of the stream is shown in the left-hand panel of Fig. 5. Focusing on the CMD of the Northern stream shown on the left-hand panel, we observe an unambiguous detection of a prominent RGB and a horizontal branch (HB) of the OS. This CMD signal can now be used to identify the likely OS member stars more efficiently as we do not expect the CMD of stream stars to vary significantly along the stream. Accordingly, we draw the mask around the main CMD features seen in the left-hand panel of Fig. 5 and select only stars lying within the mask. The mask is shown by a blue shaded region in this figure.
Fig. 6 presents the view of the OS using the GDR2 RG and BHB stars with $G<18.5$ after applying the colour-magnitude mask shown on Fig. 5 and the proper motion selection described above. The left-hand panel of the figure shows the density of the selected candidates in the stream-aligned coordinates $\phi_{1}$ and $\phi_{2}$. We only show the stream in the Northern Galactic hemisphere, as it is harder to identify in the South. The stream is readily recognizable in the region $20^{\circ}<\phi_{1}<120^{\circ}$. Below $\phi_{1} \sim 20^{\circ}$, the Galactic foreground starts to dominate and thus the stream is harder to spot despite it being closer to the Sun, while above $\phi_{1} \sim 120^{\circ}$, the stream either moves too far away, so the stellar density drops below detectable levels, or it stops altogether. Note that the estimates of the Northern extent of the stream agree well between RRL and RGBs.

To extract the track of the stream as well as its density and width, we construct a stellar density model that we fit to the data shown on the left-hand panel Fig. 6. We follow an approach similar to that adopted in Erkal, Koposov \& Belokurov (2017) to describe the Palomar 5 stream. More specifically, the properties of the stream and the background are described by cubic splines that are functions of the angle along the stream. The full model for the stellar density in a given spatial position is as follows:

$$
\begin{aligned}
\rho\left(\phi_{1}, \phi_{2}\right)= & \exp \left[\mathcal{B}\left(\phi_{1}\right)+\phi_{2} \mathcal{B}_{1}\left(\phi_{1}\right)+\phi_{2}^{2} \mathcal{B}_{2}\left(\phi_{1}\right)\right] \\
& +\exp \left[\mathcal{I}\left(\phi_{1}\right)\right] \exp \left(-\frac{1}{2}\left[\frac{\phi_{2}-\Phi_{2}\left(\phi_{1}\right)}{\exp \left[\mathcal{S}\left(\phi_{1}\right)\right]}\right]^{2}\right)
\end{aligned}
$$

where $\mathcal{B}\left(\phi_{1}\right), \mathcal{B}_{1}\left(\phi_{1}\right) \mathcal{B}_{2}\left(\phi_{1}\right), \mathcal{I}\left(\phi_{1}\right), \mathcal{S}\left(\phi_{1}\right)$, and $\Phi_{2}\left(\phi_{1}\right)$ are the splines for the logarithm of the background density, the slope of log-background across the stream, the quadratic term for the log-background, the logarithm of stream's central stellar density, the logarithm of the stream width, and stream track on the sky, respectively. The parameters of the model are the values of the spline at the spline nodes/knots. In contrast with Erkal et al. (2017), we do not adjust the location of the knots, so they are spaced equidistantly from $\phi_{1}=12.5^{\circ}$ to $121.5^{\circ}$. To model the Gaia data, we adopt a spline with seven knots for the density, five knots for the stream width, eight knots for the stream track, and seven knots for the background density and the slopes. The data used in the modelling are the number counts of stars in $1^{\circ} \times 0.1^{\circ}$ wide bins in $\phi_{1}$ and $\phi_{2}$, while the likelihood function is the Poisson likelihood for the number counts given the density model. The model is implemented in Stan probabilistic programming language 

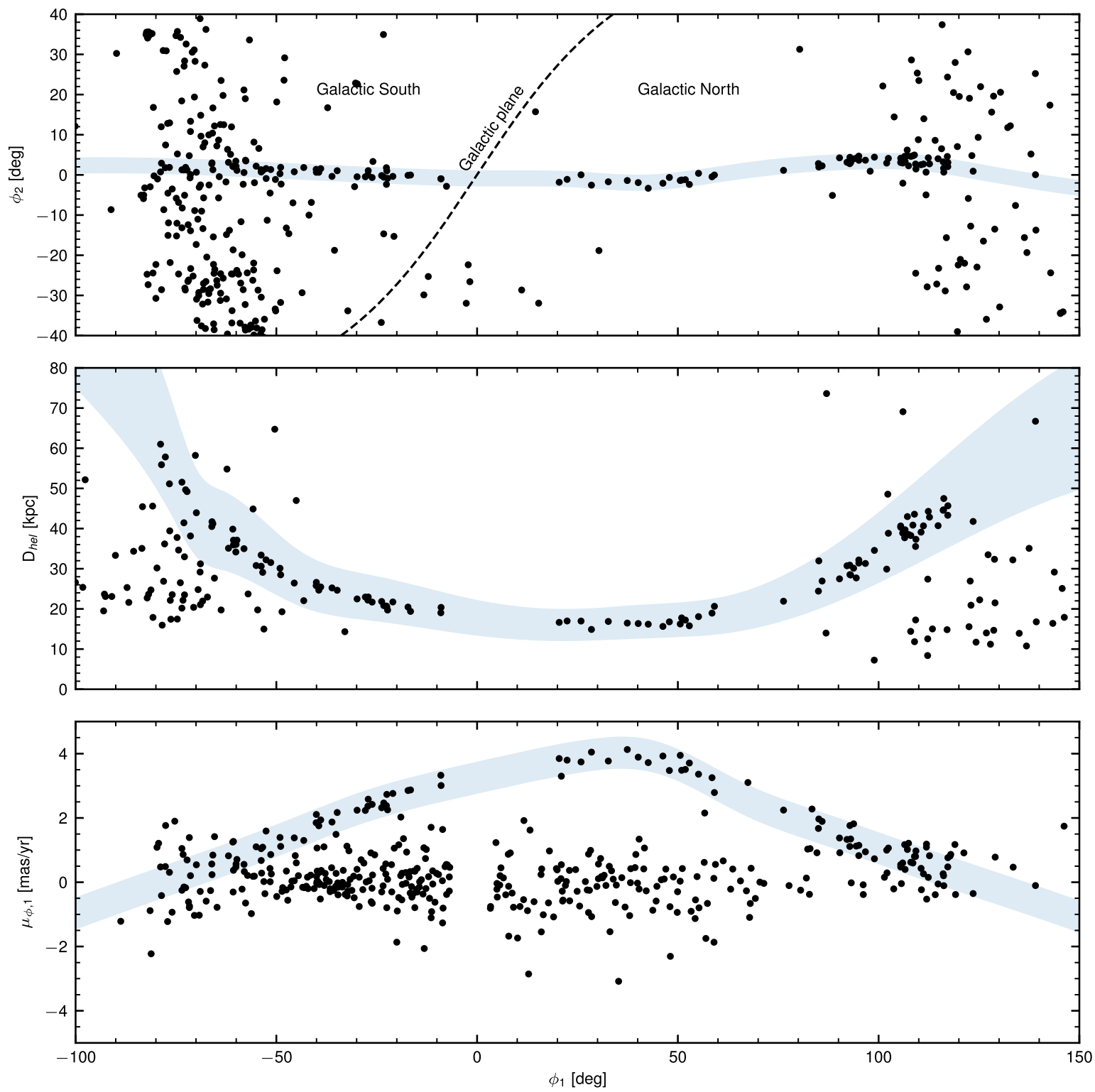

Figure 2. The selection of OS RRL in various projections of the data. Each of the panels shows the sample of stars based on the selection of stars from the other panels. The common selection criteria applied to all the panels are the AEN, parallax, and BP/RP excess cuts described in the text as well as selection based on proper motion across the stream -0.5 mas yr$^{-1}<\mu_{\phi_{2}}<0.9$ mas yr$^{-1}$. Top panel: the distribution of RRL on the sky in stream coordinates. The star selection used for this plot is based on the distance and proper motion information. The transparent blue band shows our selection region for the stream track on the sky. Middle panel: the heliocentric distance to RRL as a function of angle along the stream. The sample of stars uses the selection based on the track on the sky and proper motion (as defined in the bottom and top panels). The blue band shows the selection region for distances. Bottom panel: the proper motion along $\phi_{1}$ (with the correction for the solar motion applied) versus the angle along the stream. The sample of stars shown on the panel is based on selections shown on top and middle panels. The blue band shows the proper motion selection region.

(Carpenter et al. 2017). The only non-trivial priors used are those on the stream width values at the knots: $\mathcal{N}(\log 0.9,0.25)$ and stream track: $\mathcal{N}(0,2.5)$. The posterior of the model is sampled by Stan software using Hamiltonian Monte Carlo No-U-Turn-Sampling algorithm (Brooks et al. 2011; Hoffman \& Gelman 2014; Betancourt 2017). We use 15 parallel chains that ran for 2000 iterations each. The convergence of the chains is verified by the Gelman-Rubin $\hat{R}<1.1$ diagnostic (Gelman \& Rubin 1992). When we provide the measurements of individual parameters from the chains, those are medians with 16 percent, 84 percent error bars, or standard deviations in the case of symmetric posteriors. We provide the code of the model as Supporting Information of the paper, as well as on Github. ${ }^{1}$ The right-hand panel of Fig. 6 displays the best-fitting model representing both the stream and the Galactic foreground in the plane of $\phi_{1}$ and $\phi_{2}$. The stream model is able to reproduce the observed OS properties over the entire range of the stream longitudes considered. In particular, in both the data and the model, the stream can be seen running at a roughly constant $\phi_{2}$ for $20^{\circ}$ $<\phi_{1}<50^{\circ}$. The OS then climbs up from negative $\phi_{2}$ at $\phi_{1}$ $\sim 50^{\circ}$ to $\phi_{2} \sim 4^{\circ}$ around $\phi_{1} \sim 90^{\circ}$. From there onwards, the stream appears to broaden and gently slope down for $90^{\circ}<\phi_{1}$

${ }^{1}$ https://github.com/segasai/orphan_stream_paper 
Table 1. The subset of the likely Orphan RRL selected based on their distance, proper motion, and position on the sky. The full version of the table is available online.

\begin{tabular}{lcrc}
\hline Source_id & $\begin{array}{c}\alpha \\
(\mathrm{deg})\end{array}$ & $\begin{array}{c}\delta \\
(\mathrm{deg})\end{array}$ & $\begin{array}{c}D_{\text {hel }} \\
(\mathrm{kpc})\end{array}$ \\
\hline 38904055355908322816 & 155.36706 & 17.52019 & 32.0 \\
6417782236698088576 & 276.18759 & -74.20531 & 22.5 \\
6572607831962125056 & 329.77628 & -40.60788 & 38.2 \\
642802947461337728 & 150.64721 & 25.24754 & 30.1 \\
6561357319748782976 & 331.32574 & -46.60868 & 41.7 \\
6459293778511267200 & 321.18679 & -58.68641 & 32.2 \\
5793432594747653376 & 225.44879 & -74.39093 & 19.4 \\
6450297166352626048 & 312.65244 & -64.49243 & 26.4 \\
6564567275226088192 & 328.87316 & -46.27519 & 41.2 \\
6459507255565439744 & 321.67634 & -57.45849 & 33.4 \\
3763707471010331520 & 163.83093 & -7.17632 & 20.6 \\
799463292628940672 & 146.00854 & 36.26583 & 40.3 \\
5792708879873126400 & 227.70794 & -74.57514 & 20.5 \\
6425035538810325248 & 311.72339 & -67.05691 & 22.0 \\
& $\ldots$ & & \\
\hline
\end{tabular}

$<120^{\circ}$. Table 2 provides the stream track measurements from the model.

The stream can also be revealed and measured in the deeper DECaLS imaging. The DECaLS DR7 imaging footprint covers the stream from $50^{\circ} \lesssim \phi_{1} \lesssim 100^{\circ}$ at survey depth of $r \sim 23$. Note however, that to extract the OS signal at the magnitudes fainter than the Gaia 's limit, where the proper motion information is not available, we have to resort to a matched-filter approach (see e.g. Rockosi et al. 2002). In particular, we use the 12 Gyr PARSEC isochrone (see Marigo et al. 2017) with $[\mathrm{Fe} / \mathrm{H}]=-1.5$ and the RRL distance track to describe the colour-magnitude distribution of the stream stars. To define the best colour-magnitude filter, we split the whole DECaLS field into 100 bins of $\phi_{1}$ and construct the best binary CMD mask for a given $\phi_{1}$. We remark that, similarly to Erkal et al. (2017), we use an optimal binary CMD mask instead of using the weights - defined in Rockosi et al. (2002) - to preserve the Poisson distribution of the filtered map. Here, we use all the sources from DECaLS DR7 that were classified as PSF (type $=$ 'PSF') and have $r<23$. Fig. 7 shows the resulting stellar density distribution, where the stream can be traced over $\sim 45^{\circ}$. In this particular slice of the OS, the stream seems to be following closely a great circle without significant curvature. The vertical structure at $\phi_{1} \sim 80^{\circ}$ is the Sagittarius stream. We remark that the stellar density along the stream cannot be interpreted directly as the colour-magnitude matched filter mask is different for different $\phi_{1}$. The right-hand panel of the figure presents the spline model of the stellar density shown in the left-hand panel. The functional form for the model is the same as used for the Gaia RGB stars (equation 2) but has a different number of knots ( 7 for the stream width and intensity, 10 for the stream track, and 15 knots for the background and slopes). The measurements of the stream track are recorded in Table 3.

Fig. 8 summarizes the measured properties of the stream in different tracers as well as compares them with previous measurements. The top panel shows the stream tracks on the sky from the GDR2 RGs (solid black) and the DECaLS matched-filtered data (solid grey). Blue circles show individual GDR2 RRL selected in Section 2. It is reassuring to see that the GDR2 RG and DECaLS $\phi_{2}$ centroids of the tidal debris agree perfectly with each other and with the on-sky positions of the GDR2 RRL. Interestingly, for $70^{\circ}$ $<\phi_{1}<100^{\circ}$, both GDR2 RG and DECaLS tracks show a small offset downwards with respect to the RRL locations. However, this mismatch is modest (smaller than the stream width). In the middle panel, we show the comparison of the measured tracks with another tracer, this time BHB stars. We select these BHB stars from the SDSS using the standard $u-g, g-r$ colour criteria (Sirko et al. 2004; Deason, Belokurov \& Evans 2011), proper motion $\left|\mu_{\phi_{1}}-\hat{\mu}_{\phi, 1}\left(\phi_{1}\right)\right|<0.75,\left|\mu_{\phi_{2}}\right|<0.75$, and distance $\left|g-M_{\mathrm{G}}(g-r)-5 \log _{10}\left(D \hat{\left(\phi_{1}\right)}\right)+5\right|<0.3$ cuts (where $M_{\mathrm{G}}(g-$ $r$ ) is the colour-absolute magnitude relation from Deason et al. 2011). We note that the BHBs seem to match the other measured tracks, suggesting that the reason for the mismatch with RRL could be either some incompleteness in the catalog or random sampling effects. The bottom panel of Fig. 8 shows the measured widths from Gaia, DECaLS, and SDSS BHB stars as a function of angle along the stream. Curiously, there seems to be a slight inconsistency in the stream width behaviour. In the same region of the sky, i.e. $70^{\circ}<$ $\phi_{1}<100^{\circ}$, the DECaLS-based measurements yield a slightly wider stream than deduced from the GDR2 RG sample. The BHBs are also pointing at a higher stream width. One possible explanation for the minor inconsistencies in the stream track measurements with different stellar tracers could be the strong variation of the foreground in this portion of the sky. As Fig. 7 demonstrates, the region with $70^{\circ}<\phi_{1}<90^{\circ}$ is affected by the presence of the prominent and broad Sagittarius tidal stream. An alternative explanation is instead that the OS has different stellar sub-populations that also have different stream widths. That scenario would naturally occur if the disrupting system had components with different sizes and velocity dispersions.

\subsection{The Stream CMD}

Having clarified the distribution of the OS stars on the sky, we can now refine the stream's signal in the CMD using the combination of Gaia and DECaLS data. We use the region with $60^{\circ}<\phi_{1}<$ $98^{\circ}$ and $\left|\phi_{2}-\Phi_{2}\left(\phi_{1}\right)\right|<1^{\circ}$ to select stream stars and $60^{\circ}<$ $\phi_{1}<98^{\circ}$ and $2^{\circ}<\left|\phi_{2}-\Phi_{2}\left(\phi_{1}\right)\right|<5^{\circ}$ region for the foreground CMD. Here, the $\Phi_{2}\left(\phi_{1}\right)$ is the spline stream track model determined from DECaLS/Gaia data, rather than Gaia RRL (see Table 3). We apply proper motion selection cuts base on RRL proper motion track $\left|\mu_{\phi_{1}}-\hat{\mu}_{\phi, 1}\left(\phi_{1}\right)\right|<1$, and $\left|\mu_{\phi_{2}}\right|<1$ and shift each star by the distance modulus measured from RRL $5 \log \left(\hat{D}\left(\phi_{1}\right)\right)-5$. The stream's Hess diagram (density of stars in the CMD space) in DECam filter system is shown in Fig. 9.

While Gaia comfortably detects the stream RRL and RG stars, its photometry is too shallow to reach the MSTO at the typical distances of the OS tidal debris. The addition of the deeper DECaLS data allows us to reach the MSTO and thus break the distance-metallicity degeneracy. Dark regions (corresponding to overdense portions of the Hess diagram) in Fig. 9 reveal several familiar subsets of a codistant and coeval stellar population. The most prominent feature here is the RGB, but a tight HB is also clearly visible, attesting to the quality of the distance measurements. Less obvious, but also discernible are hints of the asymptotic giant branch and perhaps even the Blue Stragglers. Given the presence of the MSTO and with an accurate distance measurement in hand we can overlay the appropriate isochrone. As evidenced from the figure, an old and metal-poor $([\mathrm{Fe} / \mathrm{H}]=-2$, blue line $)$ isochrone is too bright for the stream's MSTO and too steep for its RGB. An isochrone with $[\mathrm{Fe} / \mathrm{H}]=-1.5$ (red line) does a better job at describing the OS stellar populations. 

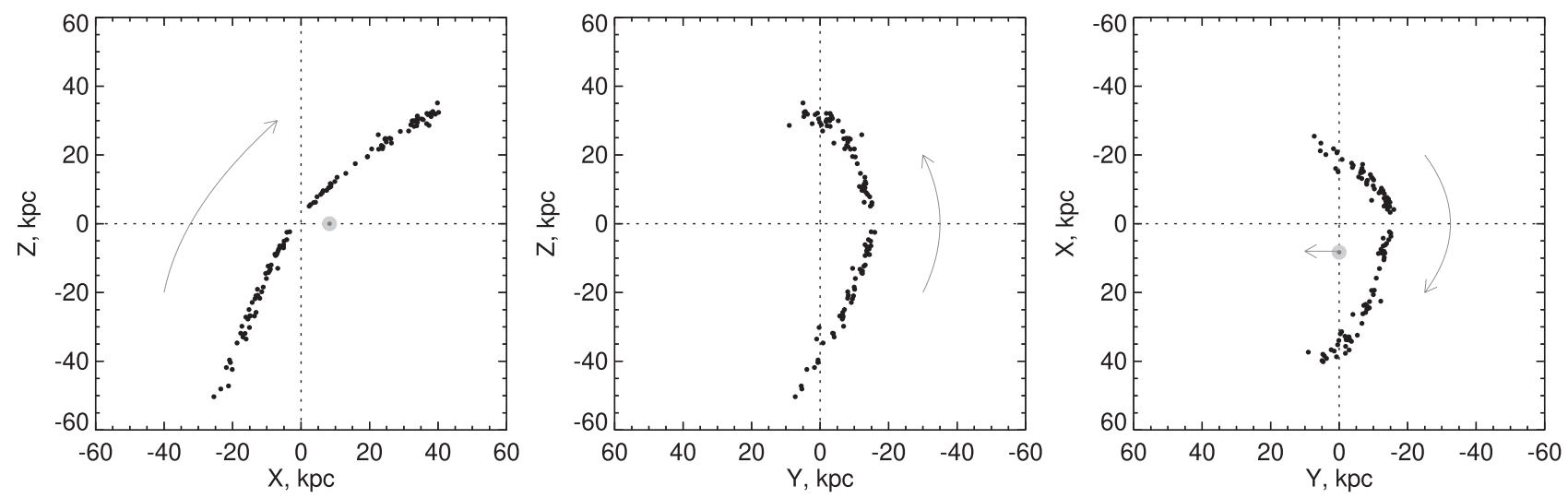

Figure 3. Same as Fig. 1, but limited to likely OS member RRL (see Fig. 2 and the main text for details). Long curved arrow shows the direction of motion of the OS, while the short straight arrow indicates the motion of the Sun (shown as grey filled circle at $(X, Y, Z)=(8.3,0,0)$.

Going back to Fig. 5, we can compare the CMDs constructed separately for the Northern (left) and Southern (right) portions of the stream. These Hess diagrams use Gaia data only and apply cuts on the star's proximity to the OS track on the sky and in the proper motion space (see previous section). Limited to the Gaia- and North-only data, the stream's CMD appears tidier than that shown in Fig. 9, albeit reaching only the bottom of the RGB. The CMD of the Southern part of the stream (righthand panel), on the other hand, looks clearly worse, most likely due to higher levels of Galactic extinction and the more severe foreground contamination. None the less, the two Hess diagrams seem to share several features in common: the brighter portions of the RGB, as well as hints of the AGB and the HB. Given the large amount of noise in the Southern OS CMD, we can only conclude that the stream stellar populations below and above the Galactic disc are roughly consistent with each other, though slight differences are still permitted by the data in hand. This motivates a spectroscopic follow-up campaign to obtain detailed chemistry for RGB stars across the full extent of the stream to place stronger limits on stellar population variations over the entire length of the OS.

\subsection{The Stream radial velocities in the SDSS}

This subsection complements the 5-D measurements of the OS track with a look at the stream's line-of-sight velocities identified in the SDSS data. We cross-match the SDSS stars with SEGUE stellar parameter pipeline (SSPP) parameter measurements (Lee et al. 2008; Allende Prieto et al. 2008; Yanny et al. 2009; Ahn et al. 2012) with the GDR2. Then, we select the likely OS member stars according to their position on the sky $\left|\phi_{2}-\Phi_{2}\left(\phi_{1}\right)\right|<1^{\circ}$ using the Gaia RGB track, proper motion $\left|\mu_{\phi}, 1-\hat{\mu}_{\phi, 1}\left(\phi_{1}\right)\right|<1$ mas yr $^{-1}$ and their position in CMD space, using the CMD mask shifted to the distance of the stream $\hat{D}\left(\phi_{1}\right)$ at each $\phi_{1}$. We also require the metallicity to be $[\mathrm{Fe} / \mathrm{H}]<-1$ and the star to be classified to be above the main sequence $\log g<4$. Top panel of Fig. 10 shows the line-of-sight velocities (corrected for the Solar reflex motion) of the likely OS stars as a function of the angle along the stream. The stream radial velocity (RV) signal is obvious and consistent with previous measurements (see e.g. Newberg et al. 2010). To take care of the contamination and noticeable uncertainties in RVs, we model this velocity distribution with a mixture of Gaussians where the behaviour of the mean stream velocity, the stream width in velocity as well as the mixing fraction of the contamination is described by splines, i.e.:

$$
\begin{aligned}
\mathcal{P}\left(V \mid \phi_{1}, \theta\right)= & f_{\mathrm{bg}}\left(\phi_{1}\right) \mathcal{N}\left(V \mid V_{\mathrm{bg}}, \sigma_{\mathrm{bg}}\right) \\
& \left.+1-f_{\mathrm{bg}}\left(\phi_{1}\right)\right) \mathcal{N}\left(V \mid V_{\mathrm{str}}\left(\phi_{1}\right), \sigma_{\mathrm{str}}\left(\phi_{1}\right)\right)
\end{aligned}
$$

here $\mathcal{N}()$ is the normal distribution and $\theta$ is the shorthand for all model parameters. $V_{\mathrm{bg}}, \sigma_{\mathrm{bg}}$ are the mean velocity and the velocity dispersion of the contaminating population (predominantly the MW stellar halo). $V_{\text {str }}\left(\phi_{1}\right), \sigma_{\text {str }}\left(\phi_{1}\right)$ are the mean velocity and the velocity dispersion of the stream. $f_{\mathrm{bg}}\left(\phi_{1}\right)$ is the fraction of the background stars that is parametrized as a logit-transform of a spline. The model is implemented in STAN and the code is provided as Supporting Information. The locations of spline knots for the velocities together with the measurements of velocities at the knots are given in Table 4. We show the measurements in the middle and bottom panels of Fig. 10. Unsurprisingly, the RV measurements display the same trend as the one discernible in the top panel of the figure. The velocity dispersion measurement, on other hand, shows roughly constant value throughout the stream of $\sim 5 \mathrm{~km} \mathrm{~s}^{-1}$. Therefore, it is likely that the increased apparent scatter in the top panel of Fig. 10 at high $\phi_{1}$ is mostly due to higher random RV errors of the fainter stream stars.

\subsection{The population of RR Lyrae stars}

In this section, we discuss the properties of the RRL population of the stream, in particular in terms of their Oosterhoff types I or II (Oosterhoff 1939), defined by the location of the stars in two separate loci in the period-amplitude diagram. The fraction of RRL of an Oosterhoff type sets apart globular clusters (GCs) and dwarf galaxies: GCs exhibit the well-known Oosterhoff dichotomy, with clusters having a vast majority of their RRL either of Oosterhoff type I (OoI) or of type II (OoII); while dwarf galaxies commonly exhibit a mix of the two types as well as intermediate-type stars (see e.g. Catelan 2009).

For the Oosterhoff classification, the light-curve period is required. Out of the 109 kinematically selected RRL OS members, only the 86 identified by the Specific Objects Study (SOS) Gaia pipeline (Clementini et al. 2019) have periods reported in the vari_rrlyrae table. Out of the remaining stars, we retrieved the periods for the 14 stars with matching bona fide ${ }^{2}$ counterparts

${ }^{2} \mathrm{PS} 1$ candidate RRL with classification scores $s_{3}>0.8$ for type ab or $s_{3}>$ $0.55 \mathrm{c}$, as suggested by Sesar et al. (2017a). 

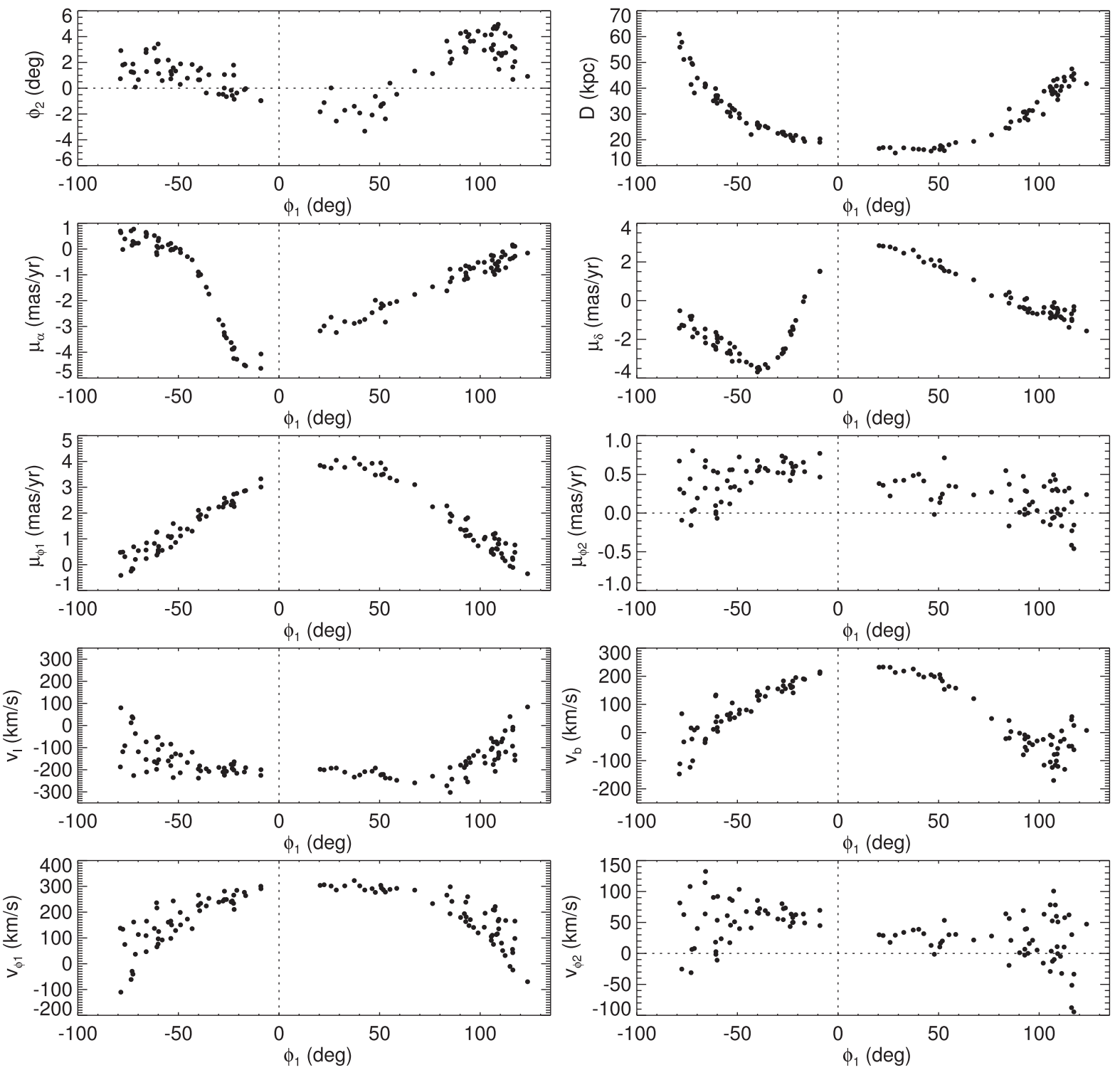

Figure 4. Phase-space projections of the likely OS member RRL as a function of the stream longitude $\phi_{1}$. Top left: positions of the OS stars on the sky in the stream-aligned coordinates $\phi_{1}, \phi_{2}$. Top right: heliocentric distance as a function of $\phi_{1}$. Second row, left: right ascension component of proper motion $\mu_{\alpha}$. Second row, right: declination component of proper motion $\mu_{\delta}$. Third row, left: proper motion along the stream (strictly speaking, along the longitude axis of the stream-aligned coordinates) $\mu_{\phi, 1}$. Third row, right: proper motion across the stream $\mu_{\phi, 2}$. Fourth row, left: velocity component along Galactic longitude $v_{1}$. Fourth row, right: velocity component along Galactic latitude $v_{\mathrm{b}}$. Bottom left: along-stream velocity $v_{\phi, 1}$. Bottom right: across-stream velocity $v_{\phi, 2}$. Note that all proper motions are corrected for the effect of the Solar reflex motion.

in the PS1 RRL catalogue from Sesar et al. (2017a). Finally, for the remaining stars we retrieved the $G, G_{\mathrm{BP}}$ and $G_{\mathrm{RP}}$ epoch photometry from the Gaia archive and obtained the light-curve periods using the Lafler \& Kinman (1965) method as adapted in Mateu et al. (2012) to consider the information in multiple bands simultaneously. Out of the nine stars for which periods were computed, we obtained good quality indicators $(\Lambda>3.5)$ for eight stars, the remaining one having too few epochs ( $<12$ per band) for a smooth light curve to be obtained with this method. The periods adopted for the RRL members are reported in Table 5 .

To compute the fraction of RRL of each Oosterhoff type, we use the period-amplitude locus given in equation (2) of Belokurov et al. (2018) to separate the stars into OoI or OoII, rescaled to convert the $V$-band amplitude of that equation to Gaia's $G$ band, using $A_{\mathrm{G}}=$
$0.925 A_{\mathrm{V}}-0.012$ as in Clementini et al. (2016). For all stars, we take range_mag_g_fov as an estimate for the amplitude. ${ }^{3}$ We do the Oosterhoff classification only for the RRL stars of type ab (84 out of 109), since the separation into Oosterhoff types is less clear for the type c (and doublemode type d) stars.

In the OS as a whole, we find 63 percent of the RRL are OoI and 37 percent are OoII. Separating the sample by Galactic latitude, we find similar fractions of 64 percent( 36 percent) and 62 per cent( 38 per cent), respectively, for OoI(OoII) in the Northern and Southern hemispheres, respectively. So, the population of RRL

${ }^{3}$ For the SOS RRL, we have checked that we get similar results using the more robust peak_to_peak_g as an amplitude estimator. 

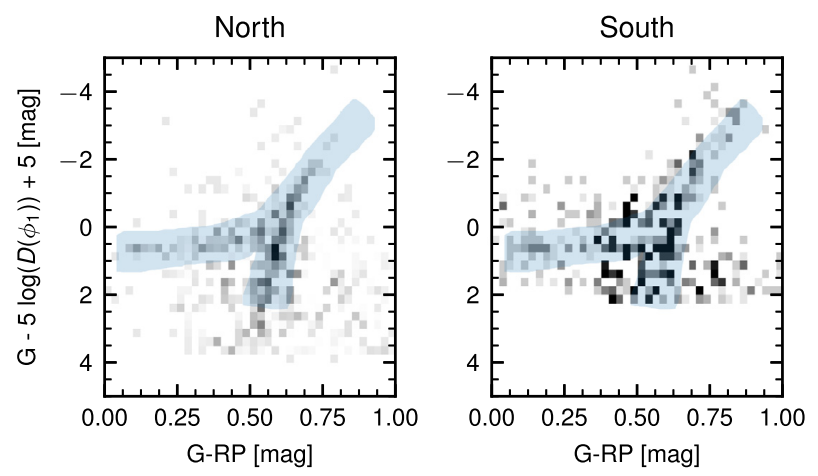

Figure 5. The Gaia background-subtracted Hess diagrams of the Northern and Southern parts of the stream. The stars have been selected based on proximity to the stream (within $0.7^{\circ}$ of the stream for the North and $1^{\circ}$ for the South) and proximity to the proper motion track $\left|\mu_{\phi_{1}}-\hat{\mu}_{\phi, 1}\left(\phi_{1}\right)\right|<$ $0.5 \mathrm{mas} \mathrm{yr}^{-1}$. The region used to determine the background Hess was $2^{\circ}<$ $\left|\phi_{2}-\hat{\phi_{2}}\left(\phi_{1}\right)\right|<4^{\circ}$ for the Northern stream and $2^{\circ}<\left|\phi_{2}-\hat{\phi}_{2}\left(\phi_{1}\right)\right|<6^{\circ}$ for the Southern. The magnitudes of all stars have been corrected by the distance modulus expected from the RRL distance track at a given $\phi_{1}$. The left-hand panel includes the data with $30^{\circ}<\phi_{1}<150^{\circ}$. The right-hand panel includes data from $-40^{\circ}<\phi_{1}<-20^{\circ}$. The blue shaded area shows the CMD mask that we adopt for further analysis of the Gaia data.

stars in the Northern and Southern parts of the stream are indistinguishable. GCs display either low $(\lesssim 0.2)$ or high ratios $(\gtrsim 0.8)$ of OoII to OoI fractions because of the Oosterhoff dichotomy, so the relatively even ratio observed $(\sim 0.6)$ indicates the RRL population resembles that of a dwarf galaxy more than that of a GC.

\subsection{Orphan Stream progenitor properties}

We can use the observed number of RRL stars in the stream to estimate the initial luminosity of the progenitor. For this, we need to first estimate how incomplete our sample might be. Comparing with the RRL from Hendel et al. (2018), we find that out of their 32 stars only 24 (75 per cent) are present in the Gaia RRL catalogue used here (prior to the kinematical selection of OS members), out which 15 are in our kinematically selected sample. The Hendel et al. (2018) sample comprises high probability RRL candidate ${ }^{4}$ members of the OS identified by Sesar et al. (2013), and, although it might also be affected by incompleteness itself, it can serve as a reference to estimate the minimum incompleteness affecting the Gaia sample. Thus, we estimate an expected number of 109 RRL of type ab, based on the 84 observed ones and assuming a (maximum) completeness of 75 per cent, in agreement with Hendel et al.'s (2018) prediction of $\sim 100$ RRL.

We use the inference model as in Mateu, Read \& Kawata (2018, see their section 4.3) based on the linear relation observed between the number of type ab RRL and the total absolute magnitude $M_{\mathrm{V}}$, for dwarf galaxies and GCs in the MW. For the 109 expected RRL of type $\mathrm{ab}$, this gives $M_{\mathrm{V}}=-10.8 \pm 1.3$, corresponding to the posterior mode and 68 per cent confidence interval, which translates into an inferred total luminosity with a most probable value at $L_{V}=3.8 \times 10^{5} L_{\odot}$ in the 68 per cent confidence interval $0.2-3 \times$ $10^{6} L_{\odot}$. The lower and upper bounds on $M_{\mathrm{V}}$ confidently place the progenitor among the classical dwarfs, with a luminosity between those of the Sextans and Leo I dwarf spheroidals (McConnachie

\footnotetext{
${ }^{4}$ And one medium probability candidate.
}

2012), with the most probable $M_{\mathrm{V}}$ placing it in the top five most luminous dwarfs, just close to Leo I. This is a robust result, that holds even if no correction for incompleteness is made, in which case $M_{\mathrm{V}}=-10.4 \pm 1.3$, i.e. brighter than Sculptor and still in the top five. Finally, note this is in agreement with our findings from the Oosterhoff types ratio, that also point toward a dwarf galaxy progenitor rather than a GC.

As a consistency check, we also use the mass-metallicity relation for Local Group galaxies from Kirby et al. (2013) to estimate a lower mass limit for the OS progenitor (assuming the OS has a lower metallicity than its host galaxy). Using $[\mathrm{Fe} / \mathrm{H}]=-1.5$ for the OS, we estimate that the progenitor had a stellar mass of approximately $4 \times 10^{6} \mathrm{M}_{\odot}$. This puts its progenitor at roughly between Leo I and Sculptor, consistent with the luminosity calculation from the number of RRL above (see also discussion in Hendel et al. 2018). We can also compare the velocity dispersion observed in the stream of $\sim 5 \mathrm{~km} \mathrm{~s}^{-1}$ (see Section 3.3) with the stellar velocity dispersion in dwarf galaxies, and that points towards somewhat less massive classical dwarf progenitors like Carina or Leo II with the velocity dispersions of $\sim 6 \mathrm{~km} \mathrm{~s}^{-1}$ (Walker et al. 2007). The luminosities of these dwarfs however are still consistent with estimates above.

\section{DISCUSSION}

We have provided an unprecedented view of the OS based on the Gaia DR2 as well as a panoply of other surveys. We have taken advantage of the GDR2 RRL; these old and metal-poor pulsating stars are relatively numerous in the OS, with an average density of $\sim 0.5$ per $\mathrm{deg}^{2}$ and can be traced over $210^{\circ}$ on the sky reaching distances of $\sim 60 \mathrm{kpc}$ from the Sun on either side of the Galactic plane. Viewed with the GDR2 RRL, the OS appears long and eccentric, piercing the MW's disc at around $10 \mathrm{kpc}$ and disappearing far into the Galactic halo, more or less in agreement with earlier studies (see e.g. Newberg et al. 2010; Sesar et al. 2013; Hendel et al. 2018). Unexpectedly, however, the OS's behaviour changes drastically in the Southern hemisphere: the Orphan's track on the sky bends, while the debris kinematics appear to exhibit strong across-stream motion.

\subsection{Orbital plane of the OS debris}

The striking twist of the OS can be comprehensibly demonstrated by tracking the direction of the vector normal to the orbital plane passing through individual portions of the stream. First, the celestial coordinates and line-of-sight distances of the likely OS RRL are converted into Galactocentric Cartesian coordinates, $X, Y, Z$. We then fit each of the three coordinates as function of the stream longitude $\phi_{1}$ with a high-order polynomial. This smooth 3-D track of the stream is split into 19 reference nodes, separated by $10^{\circ}$, of which there are 11 in the North and 8 in the South. The direction of the normal corresponding to the orientation of the orbital plane passing through the debris between each neighbouring pair of the stream nodes is then simply the cross product of their position vectors $X_{\mathrm{i}}, Y_{\mathrm{i}}, Z_{\mathrm{i}}$ and $X_{\mathrm{i}+1}, Y_{\mathrm{i}+1}, Z_{\mathrm{i}+1}$.

Fig. 11 presents the evolution of the OS debris plane in Galactocentric polar coordinates $l_{\mathrm{GC}}, b_{\mathrm{GC}}$, where the Northern sections of the stream are shown in blue and the Southern ones in red. As the figure demonstrates, the debris pole swings wildly, by some $\sim 20^{\circ}$, as the stream moves from the South to the North. Curiously, the bulk of this orbital plane wobble is in the direction of the Galactic $b_{\mathrm{GC}}$. Such an evolution of the stream's pole is inconsistent with the orbital 

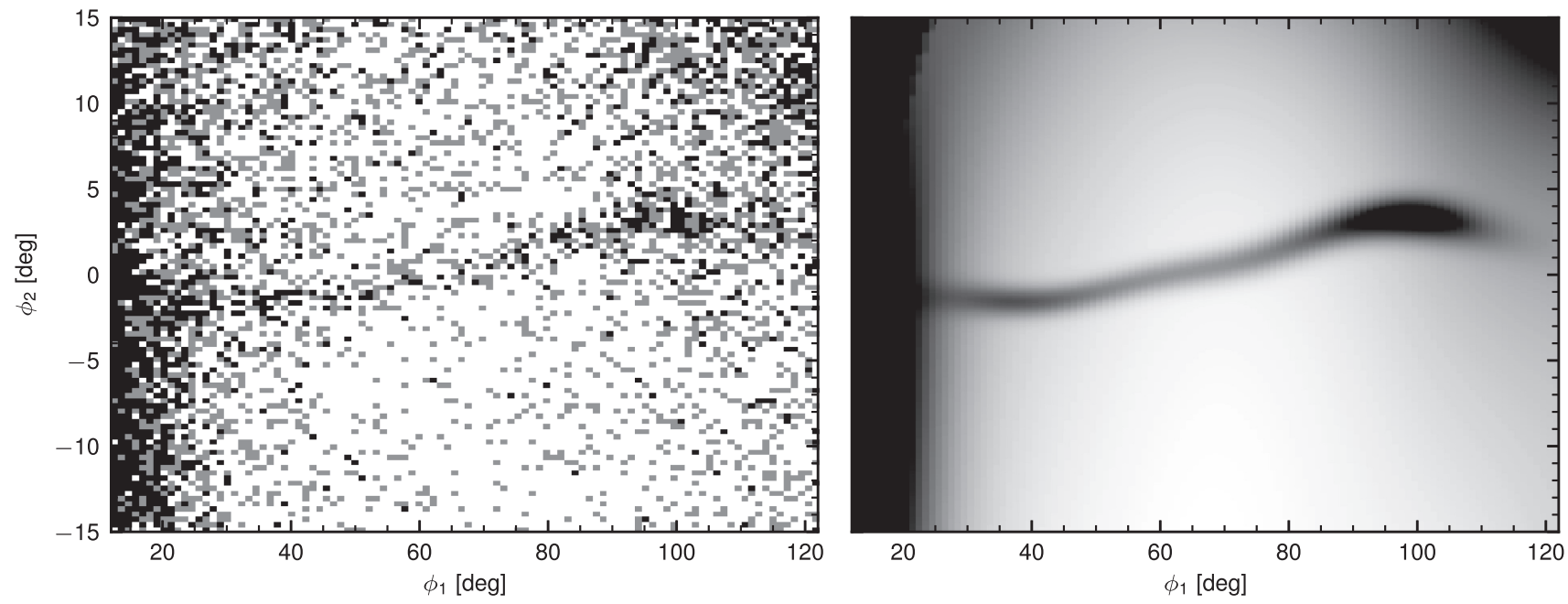

Figure 6. Left-hand panel: density distribution of Gaia RGBs in the Northern Galactic hemisphere. The stars were selected based on colour-magnitude and proper motions to be likely Orphan members. Right-hand panel: model of the stellar density including foreground and the stream.

Table 2. Stream track measurements from Gaia RGB stars.

\begin{tabular}{lcc}
\hline $\begin{array}{l}\phi_{1} \\
(\mathrm{deg})\end{array}$ & $\begin{array}{c}\phi_{2} \\
(\mathrm{deg})\end{array}$ & $\begin{array}{l}\sigma_{\phi, 2} \\
(\mathrm{deg})\end{array}$ \\
\hline 12.4 & -1.2511 & 1.7699 \\
28.0 & -1.4023 & 0.3595 \\
43.6 & -1.4896 & 0.1656 \\
59.2 & -0.2057 & 0.1936 \\
74.8 & 0.9761 & 0.1461 \\
90.4 & 2.9812 & 0.1412 \\
106.0 & 2.9882 & 0.2027 \\
121.6 & 1.4035 & 1.2939 \\
\hline
\end{tabular}

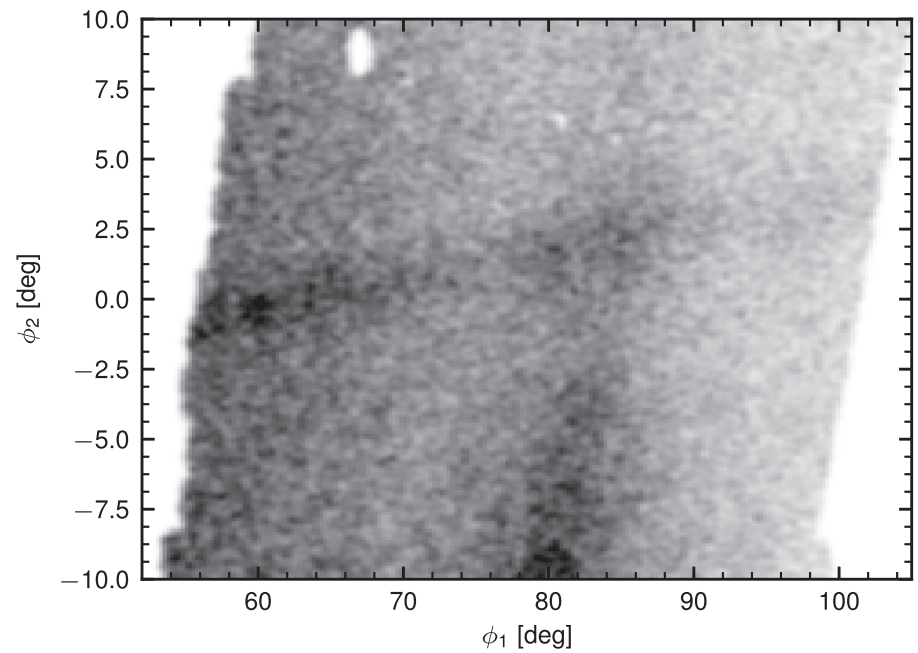

Table 3. Stream track measurements from DECaLS matched-filtered stars.

\begin{tabular}{lcc}
\hline $\begin{array}{l}\phi_{1} \\
(\mathrm{deg})\end{array}$ & $\begin{array}{c}\phi_{2} \\
(\mathrm{deg})\end{array}$ & $\begin{array}{l}\sigma_{\phi, 2} \\
(\mathrm{deg})\end{array}$ \\
\hline 53.275 & -1.6361 & 0.3234 \\
59.0194 & -0.5059 & 0.0881 \\
64.7639 & 0.1056 & 0.0955 \\
70.5083 & 0.5982 & 0.0964 \\
76.2528 & 1.203 & 0.1101 \\
81.9972 & 1.7661 & 0.0973 \\
87.7417 & 2.6617 & 0.1034 \\
93.4861 & 2.8583 & 0.1355 \\
99.2306 & 3.3051 & 0.1291 \\
104.975 & 3.5328 & 0.5728 \\
\hline
\end{tabular}

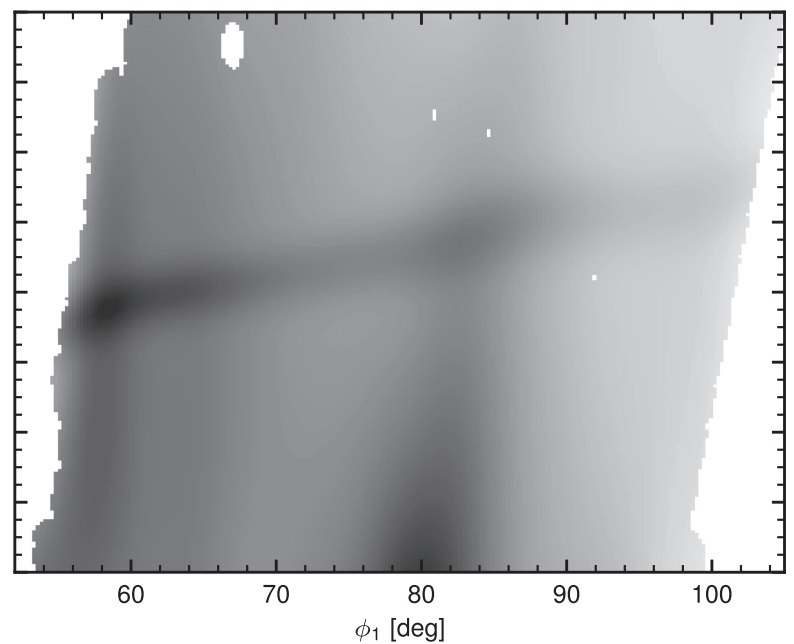

Figure 7. Left-hand panel: the stellar density of matched filter selected stars near OS. We used an old metal-poor PARSEC isochrone shifted to the distance of Orphan at each $\phi_{1}$ to define the matched filter. The OS spans the field nearly horizontally, crossing the almost vertical Sagittarius stream at $\phi_{1} \sim 80^{\circ}$. Right-hand panel: the best-fitting stellar density model of the stream. 

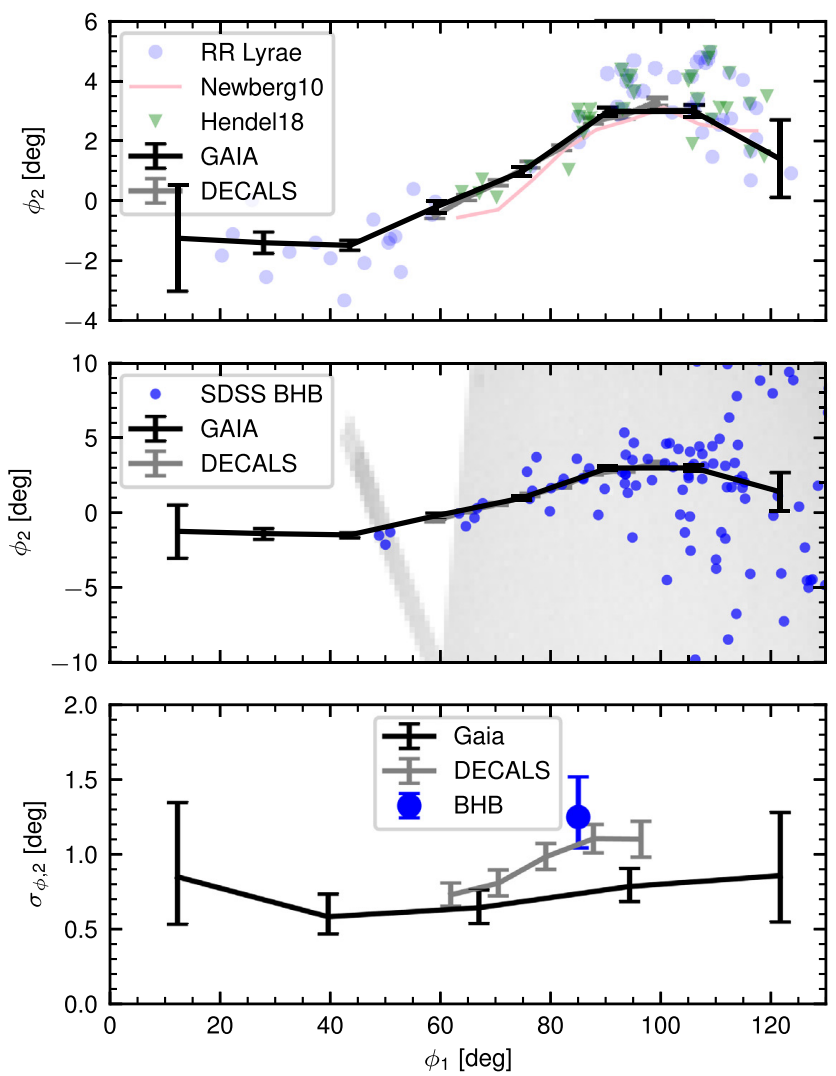

Figure 8. Measurements of the OS in the North using different tracers. Top panel: the track of OS as traced from by RRL in Gaia, RGB in Gaia, and MSTO and RGB stars in DECaLS survey. RRL are shown by blue points. The black line with error bars shows the extracted track from Gaia RGB stars. The grey line shows the stream track from DECaLS data. We also show the measurements from Newberg et al. (2009) by a pink line and potential Orphan RRL from Hendel et al. (2018) by green triangles. Middle panel: the OS as traced by BHB stars. Blue circles show BHB stars selected using SDSS $u, g, r$ photometry, proper motion, and distance. The grey shading in the background shows the SDSS footprint. We also overplot the stream tracks from DECaLS and Gaia. Bottom panel: the stream width (Gaussian $\sigma$ ) as a function of angle stream longitude, as measured from Gaia RGB stars (black line) and DECaLS (grey line) and BHBs (a single blue point).

plane precession in the potential with a flattening along the $z$-axis. As highlighted in e.g. Erkal et al. (2016a), streams in axisymmetric potentials are expected to precess around the symmetry axis. This is illustrated in Fig. 11 with two examples of the orbital pole variation, one for an orbit in an oblate (purple curve) and one in a prolate (green curve) dark matter halo. While the orbital planes do exhibit smallamplitude nutation along $b_{\mathrm{GC}}$, most of the pole displacement is in the perpendicular direction, i.e. that parallel to $l_{\mathrm{GC}}$ and around the $z$-axis. Compared to these theoretical expectations, the OS debris pole exhibits a swing with an amplitude some four times higher. We further compute the direction of the stream's angular momentum for the Northern stream, where the line-of-sight velocities are available from the SDSS data (see previous section). The direction of the stream's angular momentum and the debris pole match pretty well in the North. We expect the angular momentum pole and debris pole to decouple further down South; furthermore, the line-of-sight velocities of the Southern portions of the stream (when measured from future surveys) should manifest strong perturbations indicated by the wobble of the stream track.

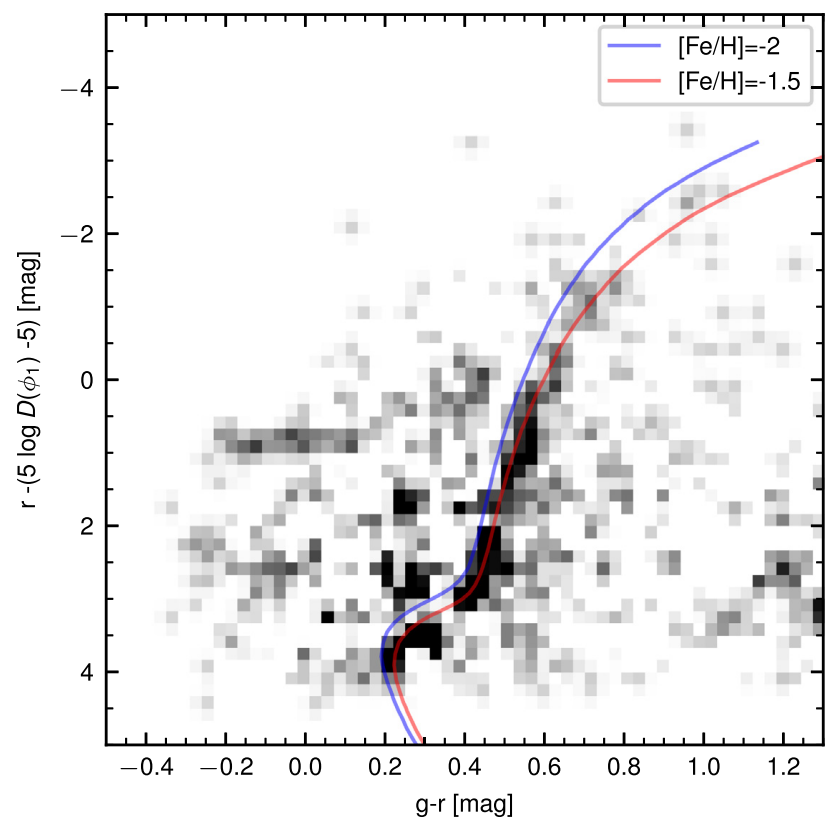

Figure 9. The background-subtracted absolute magnitude Hess diagram of the Northern OS. We use the combination of DECaLS DR7 and Gaia DR2 data. The stars were selected in the area $60^{\circ}<\phi_{1}<98^{\circ}$, based on Gaia proper motion $\left|\mu_{\phi, 1}-\hat{\mu}_{\phi, 1}\left(\phi_{1}\right)\right|<1,\left|\mu_{\phi, 2}\right|<1$ and proximity to the stream track determined from DECaLS data $\left|\phi_{2}-\Phi_{2}\left(\phi_{1}\right)\right|<1.5^{\circ}$. The background stars were selected in the region $2^{\circ}<\left|\phi_{2}-\Phi_{2}\left(\phi_{1}\right)\right|<5^{\circ}$ The magnitudes are corrected by the distance modulus model determined from RRL (see Fig. 2). We overplot two old 12 Gyr PARSEC isochrones for $[\mathrm{Fe} / \mathrm{H}]=-2$ and -1.5 .
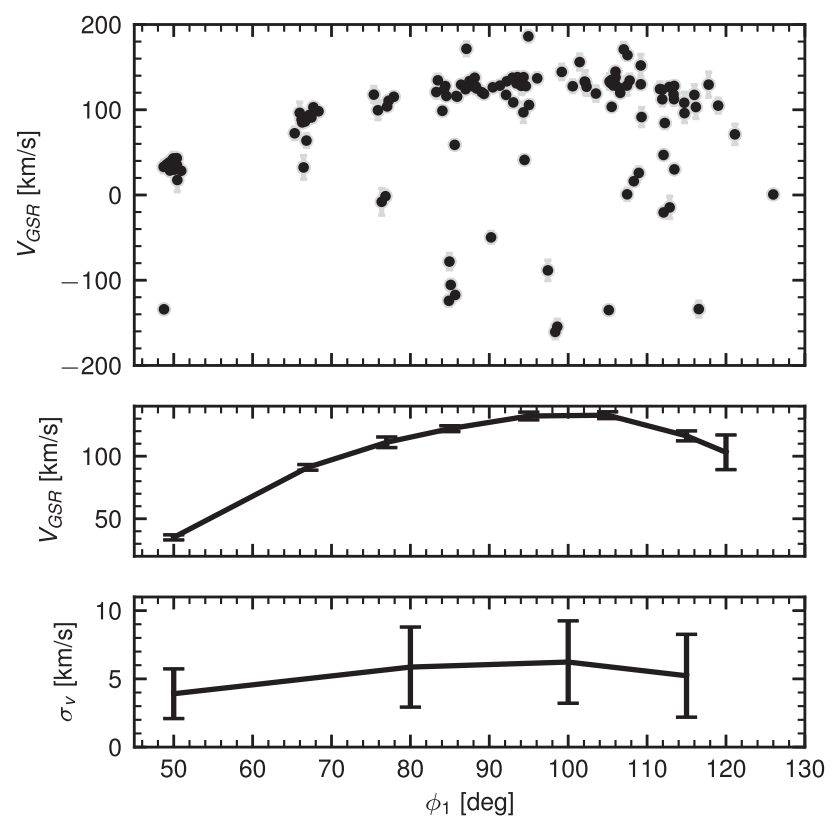

Figure 10. Top panel: heliocentric RVs of the Northern stream stars selected by the position on the sky (within $1.5^{\circ}$ of the OS track as determined from Gaia's RGBs), proper motion, and their colours and magnitudes. Note that the RVs are approaching zero near $\phi_{1} \sim 30^{\circ}$, where the heliocentric distances reach the minimum (as seen on Fig. 2) Middle panel: RV measurements extracted from the top panel using the spline model. The values and error bars show the mean and standard deviation of velocities at the spline knots. Bottom panel: the velocity dispersion measurement along the stream. The error bars are 16 per cent and 84 per cent uncertainties. 
Table 4. Measurement of OS velocity track from SDSS spectroscopic observations. The third column in the table is RV measurement uncertainty rather than velocity dispersion.

\begin{tabular}{lcc}
\hline $\begin{array}{l}\phi_{1} \\
(\mathrm{deg})\end{array}$ & $\begin{array}{c}V_{\mathrm{GSR}} \\
\left(\mathrm{km} \mathrm{s}^{-1}\right)\end{array}$ & $\begin{array}{c}\sigma_{V} \\
\left(\mathrm{~km} \mathrm{~s}^{-1}\right)\end{array}$ \\
\hline 50 & 35.097 & 2.042 \\
67 & 91.084 & 2.258 \\
77 & 111.054 & 4.072 \\
85 & 121.997 & 2.296 \\
95 & 132.123 & 3.092 \\
105 & 132.763 & 2.741 \\
115 & 116.263 & 3.964 \\
120 & 103.205 & 13.798 \\
\hline
\end{tabular}

Table 5. Adopted light-curve periods and Bailey types for the likely Orphan RRL members. The full version of the table is available online.

\begin{tabular}{lccc}
\hline Source_id & Period (d) & Type & Reference \\
\hline 3890405535908322816 & 0.772158 & RRab & $(2)$ \\
6417782236698088576 & 0.729023 & RRab & $(1)$ \\
6572607831962125056 & 0.601812 & RRab & $(1)$ \\
642802947461337728 & 0.542886 & RRab & $(2)$ \\
6561357319748782976 & 0.668472 & RRab & $(1)$ \\
6459293778511267200 & 0.590353 & RRab & $(1)$ \\
5793432594747653376 & 0.565820 & RRab & $(1)$
\end{tabular}

Notes: References: (1) = Clementini et al. (2019), (2) = Sesar et al. (2017a), and $(3)=$ this work.

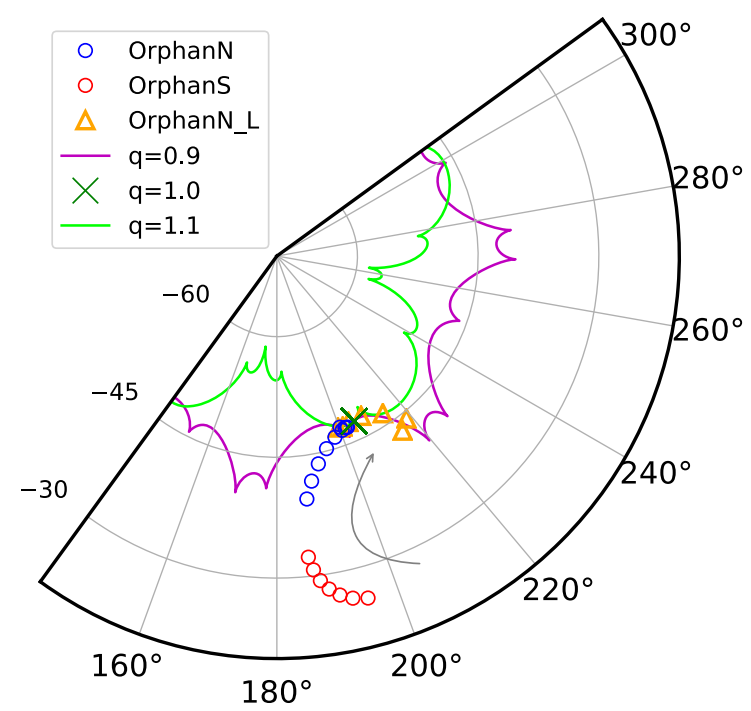

Figure 11. Galactocentric orbital pole calculated from the 3-D track of individual portions of the OS, using the positions of likely RRL stars. The Northern sections are shown in blue circles and Southern ones are in red. For part of the Northern sections where line-of-sight velocities are available from SDSS, the angular momentum pole are also calculated based on full 6-D phase-space information of RRL stars, and shown as the orange triangles. Also green and violet lines as well as the green cross show the expected orbital plane precession in a logarithmic halo potential with different flattening. The grey arrow shows the moving direction of the stream from South to North.

\subsection{Orphan Stream in the South}

At first glance, half of our stream detections appear new, as they are located under the Galactic plane. As illustrated in Fig. 12, the Southern portion of the stream crosses the footprint of the DES. On closer examination, it is clear that the Southern part of the OS is entirely consistent with the DES stream Chenab (see Shipp et al. 2018). It is likely that Chenab stars were not identified as Orphan's due to the dramatic twist in the OS track on the sky around $-20^{\circ}$ $<\phi_{1}<50^{\circ}$ and drift of the orbital pole by more than $20^{\circ}$ shown on Fig. 11. We leave the detailed analysis of Chenab-Orphan in the South for a standalone publication.

In the South, while the stream could not be easily seen with Gaia's RGBs, it is clearly visible in the DES DR1 data. Here, we proceed in the same fashion as with the DECaLS data. More precisely, we construct a set of matched filters based on the $[\mathrm{Fe} / \mathrm{H}]=-1.5,12 \mathrm{Gyr}$ PARSEC isochrone shifted to the distance $\hat{D}\left(\phi_{1}\right)$ predicted from the RRL. Fig. 13 shows the density of matched filter selected stars on the sky, with the $y$-axis being the residual offset with respect to the RRL track $\phi_{2}-\Phi_{2}\left(\phi_{1}\right)$, so that the stream is expected to go horizontally near zero. Traced with the DES data, the stream is unmistakably present, but shows more complicated morphology (compared to its Northern section). For example, the stream's signal is strongest at $\phi_{1}<-62^{\circ}$, and then drops noticeably at $\phi_{1}>-57^{\circ}$. However, in the middle of this $\phi_{1}$ interval, the debris density is greatly reduced. It is difficult to say at the moment whether this is an artefact of the data or an intrinsic variation of the stream's properties. We also note a prominent compact stellar overdensity on top the stream at $\phi_{1}$ $\sim-66^{\circ}$. This happens to be the recently discovered MW satellite Gru 2 (Drlica-Wagner et al. 2015).

\subsection{Gru 2 and other Galactic satellites}

Fig. 14 shows the position of Gru 2 (filled red star) in various phase-space projections, namely in stream-aligned coordinates $\left(\phi_{1}\right.$, $\phi_{2}$ ) (top panel), distance $\left(\phi_{1}, D\right)$ (upper middle), proper motion $\left(\phi_{1}, \mu_{\alpha}\right)$ (lower middle), and $\left(\phi_{1}, \mu_{\delta}\right)$ (bottom). Gru 2 is coincident with the OS RRL in 3 out of 4 dimensions considered: position on the sky and the two components of the proper motion. It appears offset to slightly larger distances by some $\sim 10 \mathrm{kpc}$ compared to the stream's debris at the same $\phi_{1}$. Overall, the connection between Gru 2 and the OS seems rather likely; however, line-of-sight velocity and chemical abundances are required to verify the reality and nature of that association.

Also shown in Fig. 14 are the positions of the Galactic GCs (filled circles) and ultrafaint dwarfs (UFDs; filled stars) nearest to the stream. Only seven GCs are located within $\left|\phi_{2}\right|<7^{\circ}$ of the stream's great circle. None of the globulars match well the stream's phase-space track. Of the seven objects displayed, Ruprecht 106 is closest across all dimensions, but none the less it still is considerably

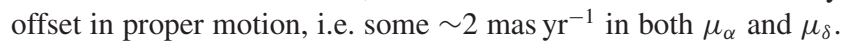
We also note that given the estimated luminosity of the Orphan progenitor galaxy (see Section 3.5), we would not expect it to host GCs. Apart from Gru 2, two additional UFDs can be seen in the vicinity of the stream, i.e. Segue 1 and UMa 2, both previously suspected to have some sort of association with OS (Fellhauer et al. 2007; Newberg et al. 2010). Interestingly, UMa 2 sits right on the continuation of the stream's track after a notable twist at $\phi_{1} \sim 100^{\circ}$. Unfortunately, as Fig. 14 demonstrates, the satellite is at least $10 \mathrm{kpc}$ closer along the line of sight and has a faster proper motion along RA. UMa 2's RV is also discrepant: according to Simon \& Geha (2007) its $V_{\mathrm{GSR}} \sim-33 \mathrm{~km} \mathrm{~s}^{-1}$, while as evident from Fig. 10, at $\phi_{1}$ 

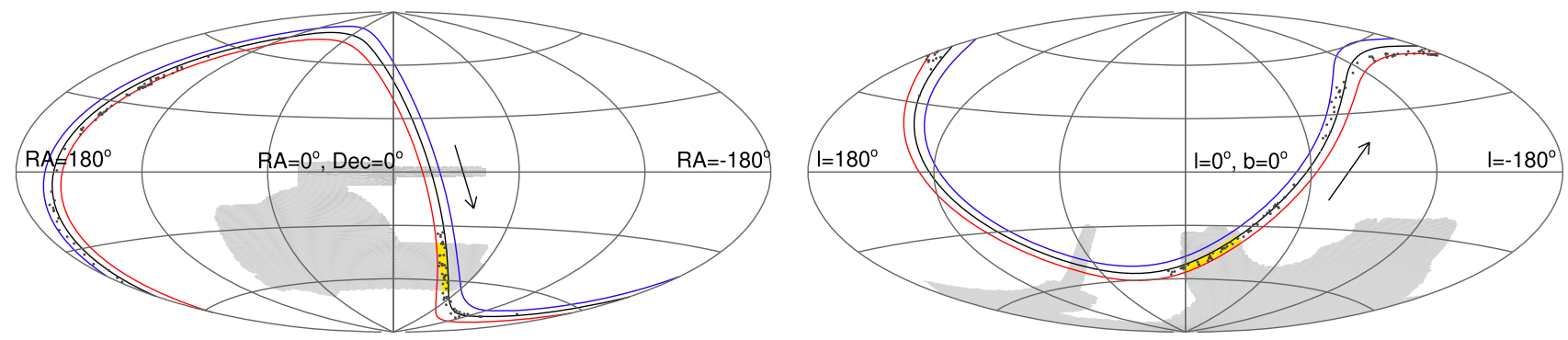

Figure 12. OS across the sky in equatorial (left) and Galactic (right) coordinates. Grey shaded area marks the boundaries of the DES DR1 footprint. Great circles parallel to the equator of the OS coordinate system and corresponding to $\phi_{2}=-4^{\circ}$ (blue), $\phi_{2}=0^{\circ}$ (black), and $\phi_{2}=4^{\circ}$ (red) are shown. Arrows indicate the direction of the stream's motion. The Chenab track as measured by Shipp et al. (2018) is shown in yellow.

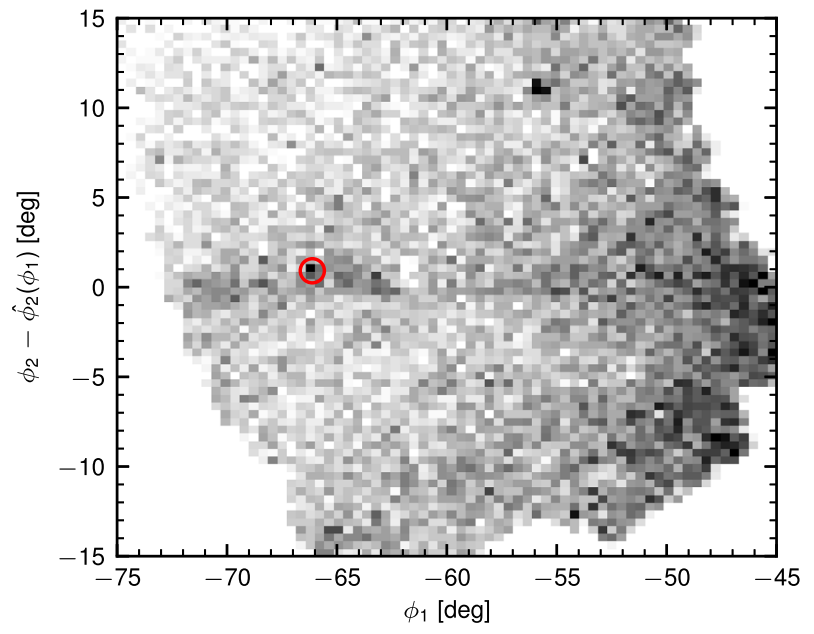

Figure 13. Density map of the OS in the South (where it was previously called Chenab). The figure shows the density of matched filter selected MSTO stars from the DES DR1 data. We used the same $\phi_{1}$-dependent matched filter based on distance, as for Fig. 7. The $y$-axis of the plot is the offset in the across the stream coordinate $\phi_{2}$ with respect to the stream track in RRL. The red circle shows the overdensity of stars associated with the Grus 2 MW satellite.

$\sim 130^{\circ}$, the OS's RV is unlikely to be lower than $50 \mathrm{~km} \mathrm{~s}^{-1}$. Segue 1 is close to the stream in distance and in $\mu_{\alpha}$ but is off by $\sim 3^{\circ}$ on the sky. More importantly, its proper motion along declination is markedly different from that of the OS's tidal debris at the same $\phi_{1}$, as first pointed out by Fritz et al. (2018). Note that there is only one classical dwarf spheroidal galaxy (Leo I) that is projected close to the stream on the sky. However, given that Leo I is located much further away, at $\sim 250 \mathrm{kpc}$, it is not shown in Fig. 14 .

\subsection{A gap in the Northern section of the Stream}

Stellar streams have been recently been put forward as excellent detectors of dark matter subhaloes (see e.g. Ibata et al. 2002; Johnston, Spergel \& Haydn 2002; Siegal-Gaskins \& Valluri 2008; Carlberg 2009; Yoon, Johnston \& Hogg 2011). If a subhalo flies near the stream, it can impart a small velocity kick to the closest stars. The affected stars change their orbits slightly, and after some time, a region near the point of the subhalo's closest approach will be evacuated, thus producing an observable stream gap (see Erkal \& Belokurov 2015). The size of such gap is linked to the properties of the perturber and the fly-by, and is directly proportional to the mass of the subhalo and the time since the interaction. Crudely, the more massive the deflector is the larger the gap. However, all gaps
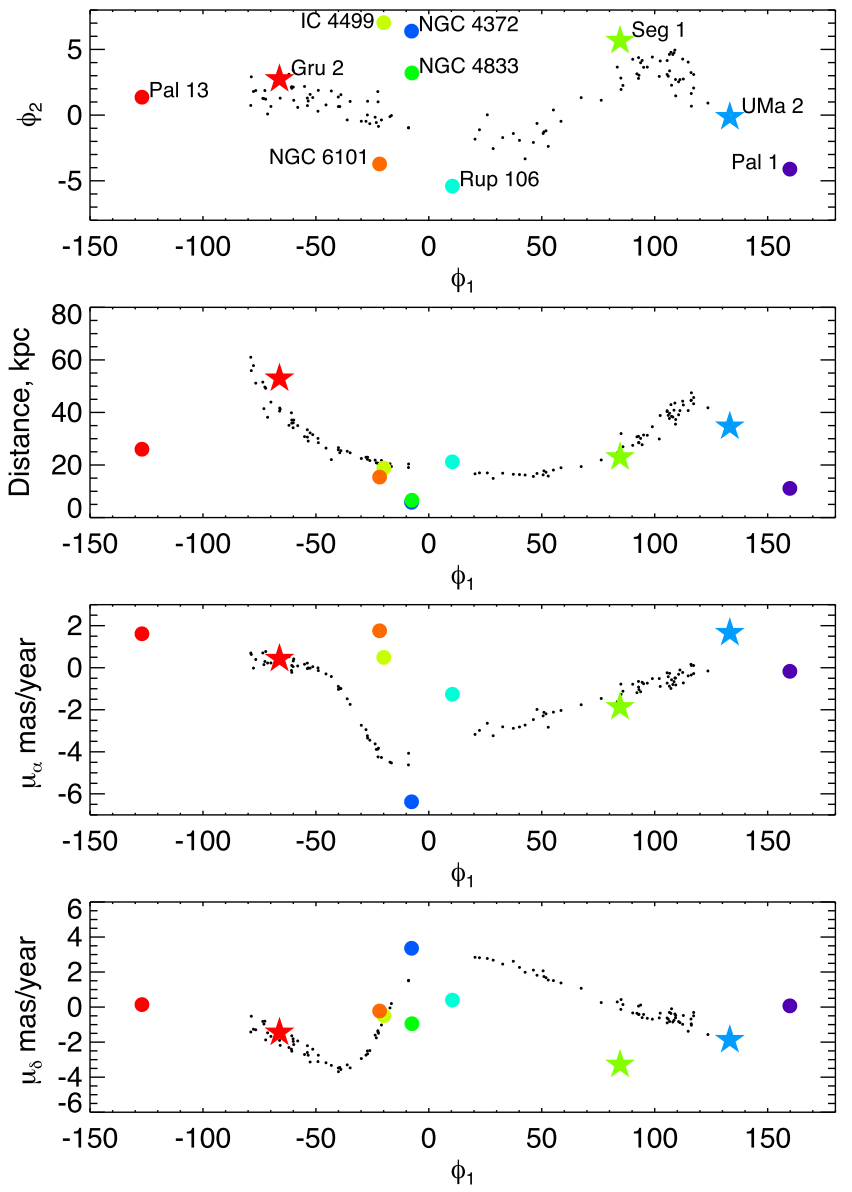

Figure 14. Phase-space coordinates of Galactic GCs (filled circles) and UFDs with $\left|\phi_{2}\right|<7^{\circ}$. GC data are from the catalogue of Vasiliev (2019), and UFD data are a combination of Simon (2018), Fritz et al. (2018), and Pace \& Li (2018). Small black dots show the positions of the OS RRL.

need an appreciable amount of time to grow in depth and hence there is a typical size scale for a detectable density depletion (see Erkal et al. 2016b). As these authors demonstrate, when interacting with streams in the MW, CMD subhaloes with masses between $10^{5}$ and $10^{9} \mathrm{M}_{\odot}$ typically produce gaps with sizes larger than $1^{\circ}$, with prevalence of gaps with $5^{\circ}-10^{\circ}$ in size.

Although Erkal et al. (2016b) focused on GC streams, these subhalo perturbations will also create gaps in dwarf galaxy streams like the OS. However, due to the larger velocity dispersion of the OS ( $\sim 5 \mathrm{~km}^{-1}$, see Section 3.3$)$, the smaller gaps will be washed 

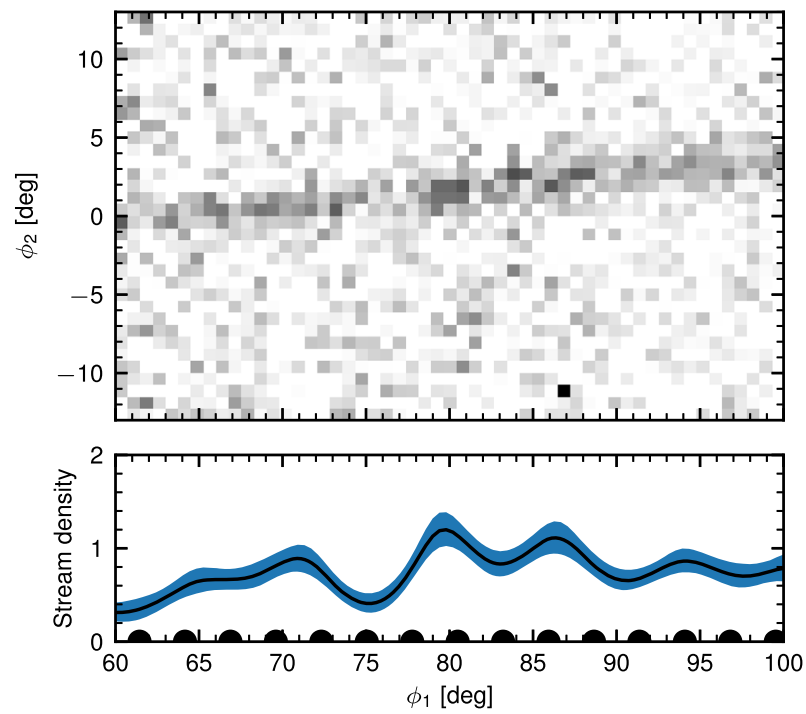

Figure 15. Top panel: the density of metal-poor MSTO stars $(0.2<g$ $-r<0.3$ and $3<M_{\mathrm{r}}<4$ in the DECaLS data, after subtracting the best-fitting background model. A quite prominent underdensity is visible at $\phi_{1} \sim 76^{\circ}$. We also notice that the stream is getting wider at higher $\phi_{1}$ confirming measurements shown on Fig. 8. Bottom panel: the results of fitting the spline model (equation 2) to the non-background subtracted MSTO density distribution. The curve and the bands are showing the median and 16 per cent, 84 per cent percentiles of the credible intervals respectively of the linear stream density from the spline model. The black circles identify the location of spline knots for the stream density. We see that the model correctly identifies the underdensity at $\phi_{1} \sim 76^{\circ}$. The stream density there drops by more than factor of $\sim 2.5$ and is highly significant at $>5 \sigma$.

out. Erkal et al. (2016b) demonstrated that significant gaps can be created by perturbations with velocity kicks down to one-tenth the velocity dispersion of the stream, corresponding to $\sim 0.5 \mathrm{~km}^{-1}$ for the OS. Assuming a typical fly-by speed of $\sim 300 \mathrm{~km} \mathrm{~s}^{-1}$, this translates to a minimum subhalo mass of $\sim 3 \times 10^{7} M_{\odot}$. Using the results of Erkal et al. (2016b), such a subhalo can create deep gaps (factor of 2 depletion) with sizes as small as several degrees.

As is clear already from e.g. Fig. 4, the number of OS RRL changes substantially as a function of stream longitude. While some of those changes could be caused by real density variations along the stream, a major factor affecting the density is potential incompleteness related to the Gaia scanning law. In fact, the region of the stream with $50^{\circ} \lesssim \phi_{1} \lesssim 90^{\circ}$ is exactly the area with the smallest number of Gaia observations, with a total number of individual light-curve points around 20, which is near the absolute minimum for the RRL identification. Therefore, we think that the paucity of RRL in this range could be explained by the Gaia catalogue incompleteness. However, our measurements of turn-off stars with DECaLS data should not suffer from these issues and should allow us to detect possible stream gaps. In fact, looking at Fig. 7 we see a hint of stream underdensity at $\phi_{1} \sim 75^{\circ}$. However, the interpretation of the figure is somewhat complicated by the use of a matched filter that varies depending on the location along the stream. Fig. 15 provides a clearer illustration of the debris density evolution in the DECaLS data. The top panel shows the density of metal-poor turn-off stars, $0.2<g-r<0.3$, within a fixed range of absolute magnitudes, $3<M_{\mathrm{r}}<4$. This image is background subtracted after fitting the MSTO density map by the spline model (see equation 2 and Section 3 for more details). The 2-D density plot shows no clear residuals of the Sagittarius stream, confirming good background subtraction and reveals a mostly well-behaved stream with the exception of the area near $\phi_{1} \sim 75^{\circ}$ where the stream density seems to noticeably drop. To assess the significance of this feature we look at the 1-D stream density profile extracted from the spline model of the MSTO distribution and shown on the bottom panel of Fig. 15. The black line and the blue band show the median and 16 per cent, 84 per cent percentiles of the stream's linear density from the posteriors of the spline model (identical to the one used in Section 3). The 1-D density profile confirms the reality of the density gap and allows us to assess its depth and significance. The stream density in the gap drops by a factor of 2.5 . According to the uncertainties on the stream density the gap is highly statistically significant $(>5.5 \sigma)$ and has a size (FWHM) of $\sim 4^{\circ}$. While it is too early to speculate about the cause of this stream gap, it is consistent with the gap created by a dark matter halo stream perturbation. We remark that given the OS pericentre is quite large $\gtrsim 15 \mathrm{kpc}$, the stream perturbation is less likely to be caused by Giant Molecular Clouds (Amorisco et al. 2016) or the bar (Hattori, Erkal \& Sanders 2016; Price-Whelan et al. 2016).

\section{CONCLUSIONS}

In this paper, we present a comprehensive all-sky view of the OS by measuring its track on the sky and in $3-\mathrm{D}$, constraining stream stars proper motions and RVs. This reveals a number of unexpected aspects of the stream's behaviour.

(i) We show that the OS is more than twice as long than previously measured, extending from the Northern Galactic hemisphere to the South with the total length of $\sim 210^{\circ}$. The stream is at its closest to the Sun at the distance of $\sim 15 \mathrm{kpc}$ when it crosses the Galactic plane and extends out to distances of $\sim 60 \mathrm{kpc}$ at the stream edges in the South and North.

(ii) As a heliocentric observer follows the stream's stars across the celestial sphere, the stream's track on the sky exhibits a clear systematic shift with an amplitude of several degrees over $210^{\circ}$ of the detected OS's length. If the distance to the debris is taken into account, the Galactocentric observer would see an even more dramatic swinging of the stream's orbital plane, i.e. of order $\sim 20^{\circ}$. This drastic debris pole evolution is mostly in the direction of Galactocentric $b_{\mathrm{GC}}$ and thus is inconsistent with precession around the $z$-axis.

(iii) Accompanying the swaying of the debris pole is the strong across-stream motion. We show that over several tens of degrees on the sky, the stream's stars appear to be moving in the direction away from the Orphan's track. Such misalignment between the debris proper motion and the stream's direction cannot arise if the stars orbit in a steady gravitational potential. We therefore hypothesize that the evolution of the debris plane and the non-zero acrossstream motion are connected and are caused by an interaction with a massive perturber.

(iv) Apart from the large-scale perturbation described above and limited to $-100^{\circ}<\phi_{1}<50^{\circ}$, a smaller amplitude twist in the stream track is visible around $\phi_{1} \sim 100^{\circ}$, i.e. at the Northern tip of the stream. Stars in this section do not show a significant streammotion misalignment and thus a different phenomenon may be needed to explain this observation.

(v) This paper demonstrates that a portion of the Southern extension of the OS had been seen previously. More precisely, a stream called Chenab was discovered in the DES data by Shipp et al. (2018) which now can be shown to match perfectly the GDR2 RRL detections discussed here. Chenab was likely not connected 
to the (previously known portion of the) OS because of the strong debris plane evolution detected in this paper.

(vi) The analysis of the total population of RRL shows that the progenitor of the stream was likely a classical dwarf galaxy with the total luminosity of $M_{\mathrm{V}} \sim-10$.

(vii) After many years of searching, a plausible connection between OS and a Galactic satellite may have been established. A UFD - Gru 2 - overlaps with the Orphan debris in most of the phase-space projections, with one small exception: along the line of sight, the satellite is $\sim 10 \mathrm{kpc}$ further away.

(viii) We identify a well-defined density gap in the OS with the size of $4^{\circ}$. The density of the stream in the gap drops by a factor of 2.5. This is consistent with the gap created by dark matter substructures with masses in the range of $\sim 10^{7}-10^{8} \mathrm{M}_{\odot}$.

This is the first paper in a series by the Orphan Aspen Treasury Collaboration. Note that, released simultaneously with this work, a companion publication (Erkal et al. 2018a) presents the results of an in-depth stream modelling exercise and provides an explanation of the observed OS properties.

\section{ACKNOWLEDGEMENTS}

This work was performed at the Aspen Center for Physics, which is supported by National Science Foundation grant PHY-1607611. We thank Michael for an illuminating discussion. The research leading to these results has received funding from the European Research Council under the European Union's Seventh Framework Programme (FP/2007-2013)/ERC grant agreement no. 308024. SK is partially supported by NSF grant AST-1813881. NK is supported by NSF CAREER award 1455260.

This work presents results from the European Space Agency (ESA) space mission Gaia. Gaia data are being processed by the Gaia Data Processing and Analysis Consortium (DPAC). Funding for the DPAC is provided by national institutions, in particular the institutions participating in the Gaia MultiLateral Agreement. The Gaia mission website is https://www.cosmos.esa.int/gaia. The Gaia archive website is https://archives.esac.esa.int/gaia.

This project used public archival data from the DES. Funding for the DES Projects has been provided by the U.S. Department of Energy, the U.S. National Science Foundation, the Ministry of Science and Education of Spain, the Science and Technology Facilities Council of the United Kingdom, the Higher Education Funding Council for England, the National Center for Supercomputing Applications at the University of Illinois at Urbana-Champaign, the Kavli Institute of Cosmological Physics at the University of Chicago, the Center for Cosmology and Astro-Particle Physics at the Ohio State University, the Mitchell Institute for Fundamental Physics and Astronomy at Texas A\&M University, Financiadora de Estudos e Projetos, Fundação Carlos Chagas Filho de Amparo à Pesquisa do Estado do Rio de Janeiro, Conselho Nacional de Desenvolvimento Científico e Tecnológico and the Ministério da Ciência, Tecnologia e Inovação, the Deutsche Forschungsgemeinschaft, and the Collaborating Institutions in the DES. The Collaborating Institutions are Argonne National Laboratory, the University of California at Santa Cruz, the University of Cambridge, Centro de Investigaciones Energéticas, Medioambientales y TecnológicasMadrid, the University of Chicago, University College London, the DES-Brazil Consortium, the University of Edinburgh, the Eidgenössische Technische Hochschule (ETH) Zürich, Fermi National Accelerator Laboratory, the University of Illinois at UrbanaChampaign, the Institut de Ciències de l'Espai (IEEC/CSIC), the Institut de Física d'Altes Energies, Lawrence Berkeley National
Laboratory, the Ludwig-Maximilians Universität München and the associated Excellence Cluster Universe, the University of Michigan, the National Optical Astronomy Observatory, the University of Nottingham, The Ohio State University, the OzDES Membership Consortium, the University of Pennsylvania, the University of Portsmouth, SLAC National Accelerator Laboratory, Stanford University, the University of Sussex, and Texas A\&M University. Based in part on observations at Cerro Tololo Inter-American Observatory, National Optical Astronomy Observatory, which is operated by the Association of Universities for Research in Astronomy (AURA) under a cooperative agreement with the National Science Foundation.

The Legacy Surveys consist of three individual and complementary projects: the DECaLS (NOAO Proposal ID no. 2014B0404; PIs: David Schlegel and Arjun Dey), the Beijing-Arizona Sky Survey (BASS; NOAO Proposal ID no. 2015A-0801; PIs: Zhou Xu and Xiaohui Fan), and the Mayall z-band Legacy Survey (MzLS; NOAO Proposal ID no. 2016A-0453; PI: Arjun Dey). DECaLS, BASS, and MzLS together include data obtained, respectively, at the Blanco telescope, Cerro Tololo Inter-American Observatory, National Optical Astronomy Observatory (NOAO); the Bok telescope, Steward Observatory, University of Arizona; and the Mayall telescope, Kitt Peak National Observatory, NOAO. The Legacy Surveys project is honored to be permitted to conduct astronomical research on Iolkam Du'ag (Kitt Peak), a mountain with particular significance to the Tohono O'odham Nation.

NOAO is operated by the Association of Universities for Research in Astronomy (AURA) under a cooperative agreement with the National Science Foundation.

The Legacy Survey team makes use of data products from the Near-Earth Object Wide-field Infrared Survey Explorer (NEOWISE), which is a project of the Jet Propulsion Laboratory/California Institute of Technology. NEOWISE is funded by the National Aeronautics and Space Administration.

The Legacy Surveys imaging of the DESI footprint is supported by the Director, Office of Science, Office of High Energy Physics of the U.S. Department of Energy under Contract No. DE-AC0205CH1123, by the National Energy Research Scientific Computing Center, a DOE Office of Science User Facility under the same contract; and by the U.S. National Science Foundation, Division of Astronomical Sciences under contract no. AST-0950945 to NOAO.

\section{REFERENCES}

Abbott T. M. C. et al., 2018, ApJS, 239, 18

Ahn C. P. et al., 2012, ApJS, 203, 21

Aihara H. et al., 2011, ApJS, 193, 29

Alam S. et al., 2015, ApJS, 219, 12

Allende Prieto C. et al., 2008, AJ, 136, 2070

Amorisco N. C., Gómez F. A., Vegetti S., White S. D. M., 2016, MNRAS, 463, L17

Balbinot E. et al., 2016, ApJ, 820, 58

Belokurov V. et al., 2006, ApJ, 642, L137

Belokurov V. et al., 2007, ApJ, 658, 337

Belokurov V. A., Erkal D., 2019, MNRAS, 482, L9

Belokurov V., Deason A. J., Koposov S. E., Catelan M., Erkal D., Drake A J., Evans N. W., 2018, MNRAS, 477, 1472

Bernard E. J. et al., 2016, MNRAS, 463, 1759

Betancourt M., 2017, preprint (arXiv:1701.02434)

Bonaca A., Geha M., Kallivayalil N., 2012, ApJ, 760, L6

Bovy J., 2015, ApJS, 216, 29

Brooks S., Gelman A., Jones G., Meng X., 2011, in Handbook of Markov Chain Monte Carlo. Chapman \& Hall/CRC Handbooks of Modern Statistical Methods. CRC Press, Boca Raton, FL 
Carlberg R. G., 2009, ApJ, 705, L223

Carpenter B. et al., 2017, J. Stat. Softw., Art., 76, 1

Casey A. R., Da Costa G., Keller S. C., Maunder E., 2013, ApJ, 764, 39

Casey A. R., Keller S. C., Da Costa G., Frebel A., Maunder E., 2014, ApJ, 784, 19

Catelan M., 2009, Ap\&SS, 320, 261

Chambers K. C. et al., 2016, preprint (arXiv:1612.05560)

Chen B. et al., 2001, ApJ, 553, 184

Clementini G. et al., 2016, A\&A, 595, A133

Clementini G. et al., 2019, A\&A, 622, A60

Dark Energy Survey Collaboration et al., 2016, MNRAS, 460, 1270

Deason A. J., Belokurov V., Evans N. W., 2011, MNRAS, 416, 2903

Deason A. J., Belokurov V., Koposov S. E., Rockosi C. M., 2014, ApJ, 787, 30

Dey A. et al., 2018, preprint (arXiv:1804.08657)

Drlica-Wagner A. et al., 2015, ApJ, 813, 109

Erkal D. et al., 2018a, preprint (arXiv:1812.08192)

Erkal D. et al., 2018b, MNRAS, 481, 3148

Erkal D., Belokurov V., 2015, MNRAS, 450, 1136

Erkal D., Sanders J. L., Belokurov V., 2016a, MNRAS, 461, 1590

Erkal D., Belokurov V., Bovy J., Sanders J. L., 2016b, MNRAS, 463, 102

Erkal D., Koposov S. E., Belokurov V., 2017, MNRAS, 470, 60

Evans D. W. et al., 2018, A\&A, 616, A4

Fellhauer M. et al., 2007, MNRAS, 375, 1171

Fermani F., Schönrich R., 2013, MNRAS, 430, 1294

Fritz T. K., Lokken M., Kallivayalil N., Wetzel A., Linden S. T., Zivick P., Tollerud E. J., 2018, ApJ, 860, 164

Gaia Collaboration et al., 2016, A\&A, 595, A1

Gaia Collaboration et al., 2018a, A\&A, 616, A1

Gaia Collaboration et al., 2018b, A\&A, 616, A10

Gelman A., Rubin D. B., 1992, Statist. Sci., 7, 457

Gillessen S., Eisenhauer F., Fritz T. K., Bartko H., Dodds-Eden K., Pfuhl O., Ott T., Genzel R., 2009, ApJ, 707, L114

Grillmair C. J., 2006, ApJ, 645, L37

Grillmair C. J., 2009, ApJ, 693, 1118

Grillmair C. J., Dionatos O., 2006, ApJ, 643, L17

Grillmair C. J., Hetherington L., Carlberg R. G., Willman B., 2015, ApJ, 812, L26

Gunn J. E. et al., 1998, AJ, 116, 3040

Hattori K., Erkal D., Sanders J. L., 2016, MNRAS, 460, 497

Helmi A., White S. D. M., 1999, MNRAS, 307, 495

Hendel D. et al., 2018, MNRAS, 479, 570

Hoffman M. D., Gelman A., 2014, J. Mach. Learn. Res., 15, 1593

Holl B. et al., 2018, A\&A, 618, A30

Ibata R., Lewis G. F., Irwin M., Totten E., Quinn T., 2001, ApJ, 551, 294

Ibata R. A., Lewis G. F., Irwin M. J., Quinn T., 2002, MNRAS, 332, 915

Ibata R. A., Malhan K., Martin N. F., Starkenburg E., 2018, ApJ, 865, 85

Iorio G., Belokurov V., 2019, MNRAS, 482, 3868

Jin S., Lynden-Bell D., 2007, MNRAS, 378, L64

Johnston K. V., Hernquist L., Bolte M., 1996, ApJ, 465, 278

Johnston K. V., Sigurdsson S., Hernquist L., 1999, MNRAS, 302, 771

Johnston K. V., Spergel D. N., Haydn C., 2002, ApJ, 570, 656

Kirby E. N., Cohen J. G., Guhathakurta P., Cheng L., Bullock J. S., Gallazzi A., 2013, ApJ, 779, 102

Koposov S. E. et al., 2012, ApJ, 750, 80

Koposov S. E., Rix H.-W., Hogg D. W., 2010, ApJ, 712, 260

Koposov S. E., Irwin M., Belokurov V., Gonzalez-Solares E., Yoldas A. K., Lewis J., Metcalfe N., Shanks T., 2014, MNRAS, 442, L85

Koppelman H., Helmi A., Veljanoski J., 2018, ApJ, 860, L11

Kuhn J. R., 1993, ApJ, 409, L13

Lafler J., Kinman T. D., 1965, ApJS, 11, 216

Lee Y. S. et al., 2008, AJ, 136, 2022

Lynden-Bell D., 1982, The Observatory, 102, 202

Lynden-Bell D., Lynden-Bell R. M., 1995, MNRAS, 275, 429

Majewski S. R., Skrutskie M. F., Weinberg M. D., Ostheimer J. C., 2003, ApJ, 599, 1082

Malhan K., Ibata R. A., 2018, MNRAS, 477, 4063

Marigo P. et al., 2017, ApJ, 835, 77
Mateu C., Vivas A. K., Downes J. J., Briceño C., Zinn R., Cruz-Diaz G., 2012, MNRAS, 427, 3374

Mateu C., Read J. I., Kawata D., 2018, MNRAS, 474, 4112

McConnachie A. W., 2012, AJ, 144, 4

Murali C., Dubinski J., 1999, AJ, 118, 911

Myeong G. C., Evans N. W., Belokurov V., Amorisco N. C., Koposov S. E., 2018, MNRAS, 475, 1537

Newberg H. J. et al., 2002, ApJ, 569, 245

Newberg H. J., Yanny B., Willett B. A., 2009, ApJ, 700, L61

Newberg H. J., Willett B. A., Yanny B., Xu Y., 2010, ApJ, 711, 32

Odenkirchen M. et al., 2001, ApJ, 548, L165

Oosterhoff P. T., 1939, The Observatory, 62, 104

Pace A. B., Li T. S., 2018, preprint (arXiv:1806.02345)

Price-Whelan A. M., Bonaca A., 2018, ApJ, 863, L20

Price-Whelan A. M., Johnston K. V., Valluri M., Pearson S., Küpper A. H. W., Hogg D. W., 2016, MNRAS, 455, 1079

Rockosi C. M. et al., 2002, AJ, 124, 349

Sales L. V. et al., 2008, MNRAS, 389, 1391

Schönrich R., Binney J., Dehnen W., 2010, MNRAS, 403, 1829

Sesar B. et al., 2013, ApJ, 776, 26

Sesar B. et al., 2017a, AJ, 153, 204

Sesar B., Fouesneau M., Price-Whelan A. M., Bailer-Jones C. A. L., Gould A., Rix H.-W., 2017b, ApJ, 838, 107

Shipp N. et al., 2018, ApJ, 862, 114

Siegal-Gaskins J. M., Valluri M., 2008, ApJ, 681, 40

Simon J. D., 2018, ApJ, 863, 89

Simon J. D., Geha M., 2007, ApJ, 670, 313

Sirko E. et al., 2004, AJ, 127, 899

Sohn S. T. et al., 2016, ApJ, 833, 235

The Dark Energy Survey Collaboration, 2005, preprint (astro-ph/0510346)

Torrealba G. et al., 2018, preprint (arXiv:1811.04082)

Vasiliev E., 2019, MNRAS, 484, 2832

Walker M. G., Mateo M., Olszewski E. W., Gnedin O. Y., Wang X., Sen B., Woodroofe M., 2007, ApJ, 667, L53

Yanny B. et al., 2009, AJ, 137, 4377

Yoon J. H., Johnston K. V., Hogg D. W., 2011, ApJ, 731, 58

York D. G. et al., 2000, AJ, 120, 1579

\section{SUPPORTING INFORMATION}

Supplementary data are available at MNRAS online.

Table 1. The subset of the likely Orphan RRL selected based on their distance, proper motion, and position on the sky.

Table 5. Adopted light-curve periods and Bailey types for the likely Orphan RRL members.

Please note: Oxford University Press is not responsible for the content or functionality of any supporting materials supplied by the authors. Any queries (other than missing material) should be directed to the corresponding author for the article.

\section{APPENDIX A: RR LYRAE QUERY}

To select the RRL used in this paper, we combined the information from two Gaia classifiers, one is provided in the vari_rrylae table and another one in the vari_classifier_result. We list the specific query below.

WITH $x$ AS (

SELECT Vari_classifier_result.source_id

FROM gaia_dr2.vari_classifier_result

WHERE

vari_classifier_result.best_class_name : : text 'RR UNION

SELECT vari_rrlyrae.source_id

FROM gaia_dr2.vari_rrlyrae

) , 
Y AS (

SELECT r.best_classification,

r.solution_id,

x.source_id, r.pf, r.pf_error,

r.p1_o, r.p1_o_error,

r.epoch-g,

r.epoch-g_error,

r.epoch_bp,

r.epoch_bp_error,

r.epoch_rp,

r.epoch_rp_error,

$r$.int_average_g,

r.int_average_g_error,

$r$. int_average_bp,

r. int_average_bp_error,

$r$.int_average_rp,

r.int_average_rp_error,

r.peak_to_peak_g,

r.peak_to_peak_g_error,

r.peak_to_peak_bp,

$r$.peak_to_peak_bp_error,

$r$.peak_to_peak_rp,

$r$.peak_to_peak_rp_error,

r.num_clean_epochs_g,

r.num_clean_epochs_bp,

r.num_clean_epochs_rp,

FROM $\mathrm{x}$

LEFT JOIN gaia_dr2, vari_rrlyrae $r$

USING (source_id)

) ,

$z$ AS (

SELECT y.*

vr.classifier_name,

vr.best_class_name,

vr.best_class_score

FROM Y

LEFT JOIN gaia_dr2.vari_classifier_result vr

ON y.source_id = vr.source_id

)

SELECT z.best_classification,

$\mathrm{z} . *, \mathrm{VV} \cdot *$

FROM $z$

LEFT JOIN gaia_dr2.vari_time_series_statistics VV

ON z.source_id = vV.source_id;

\section{APPENDIX B: TRANSFORMATION OF EQUATORIAL COORDINATES TO STREAM COORDINATES $\phi_{1}, \phi_{2}$}

$$
\begin{aligned}
& \left(\begin{array}{c}
\cos \left(\phi_{1}\right) \cos \left(\phi_{2}\right) \\
\sin \left(\phi_{1}\right) \cos \left(\phi_{2}\right) \\
\sin \left(\phi_{2}\right)
\end{array}\right) \\
& =\left(\begin{array}{ccc}
-0.44761231 & -0.08785756 & -0.88990128 \\
-0.84246097 & 0.37511331 & 0.38671632 \\
0.29983786 & 0.92280606 & -0.2419219
\end{array}\right) \\
& \quad \times\left(\begin{array}{c}
\cos (\alpha) \cos (\delta) \\
\sin (\alpha) \cos (\delta) \\
\sin (\delta)
\end{array}\right)
\end{aligned}
$$

\section{APPENDIX C: THE DEFINITION OF SPLINES USED FOR THE SELECTION OF RR LYRAE}

Table C1. The location of points defining the natural spline for the stream track shown on Fig. 2 to select RRL.

\begin{tabular}{lc}
\hline $\begin{array}{l}\phi_{1} \\
(\mathrm{deg})\end{array}$ & $\begin{array}{c}\phi_{2} \\
(\mathrm{deg})\end{array}$ \\
\hline-105.186 & 2.315 \\
-74.7184 & 2.148 \\
-54.4067 & 1.2797 \\
-25.4375 & -0.0562 \\
0.2019 & -0.8577 \\
25.8413 & -1.1917 \\
39.1605 & -1.9598 \\
66.9643 & 1.1127 \\
93.1032 & 3.2835 \\
108.0872 & 3.2167 \\
129.8974 & -0.1564 \\
162.5293 & -4.8653 \\
\hline
\end{tabular}

Table C2. The location of points defining the natural spline for the heliocentric distance track shown on Fig. 2 to select RRL.

\begin{tabular}{lc}
\hline $\begin{array}{l}\phi_{1} \\
(\mathrm{deg})\end{array}$ & $\begin{array}{c}\text { Heliocentric distance } \\
(\mathrm{kpc})\end{array}$ \\
\hline-100.0 & 100.0 \\
-76.2169 & 58.0 \\
-69.9684 & 44.0 \\
-63.1307 & 39.441 \\
-44.2712 & 27.6252 \\
-25.0268 & 22.5353 \\
-5.3975 & 18.7178 \\
20.0052 & 15.9911 \\
42.3287 & 16.9 \\
61.958 & 18.5361 \\
90.0548 & 29.2612 \\
114.3027 & 44.8945 \\
\hline
\end{tabular}

Table C3. The location of points defining the spline for the track of proper motion along the stream shown on Fig. 2 to select RRL.

\begin{tabular}{lc}
\hline $\begin{array}{l}\phi_{1} \\
(\mathrm{deg})\end{array}$ & $\begin{array}{c}\mu_{\phi, 1} \\
\left(\mathrm{mas} \mathrm{yr}^{-1}\right)\end{array}$ \\
\hline-77.4458 & 0.0408 \\
-49.626 & 1.3103 \\
-28.3432 & 2.3325 \\
-2.1956 & 3.1889 \\
24.2559 & 3.881 \\
44.0186 & 3.9108 \\
65.9096 & 2.6219 \\
88.4086 & 1.569 \\
103.6106 & 0.8984 \\
124.7415 & 0.0051 \\
\hline
\end{tabular}

This paper has been typeset from a $\mathrm{T}_{\mathrm{E}} \mathrm{X} / \mathrm{LT} \mathrm{E} \mathrm{X}$ file prepared by the author. 